\title{
Remote Triggering Not Evident Near Epicenters of Impending Great Earthquakes
}

\author{
by Nicholas J. van der Elst, ${ }^{*}$ Emily E. Brodsky, and Thorne Lay
}

\begin{abstract}
Recently, there have been numerous great $\left(M_{\mathrm{w}} \geq 8\right)$, devastating earthquakes, with a rate in the last seven years that is $260 \%$ of the average rate during the 111-year seismological history. Each great earthquake presents an opportunity to study a major fault at the very beginning and end of the inferred seismic cycle. In this work, we use these events as both targets and sources to probe susceptibility to dynamic triggering in the epicentral region before and after a large earthquake. This study also carefully addresses the possibility that large earthquakes interact in a cascade of remotely triggered sequences that culminates in further large earthquakes. We seek evidence of triggering associated with the 16 great $M_{\mathrm{w}} \geq 8$ events that occurred between 1998 and 2011, using regional and global earthquake catalogs to measure changes in interevent time statistics. Statistical significance is calculated with respect to a nonstationary reference model that includes mainshock-aftershock clustering. We find limited evidence that a few great earthquakes triggered an increase in seismicity at the site of the next great earthquake in the sequence. However, this evidence is not corroborated by all statistical tests nor all earthquake catalogs. Systematic triggered rate changes in the years to decades before each great earthquake are less than $19 \%$ at the $95 \%$ confidence level, too small to explain the observed rate increase. The catalogs are insufficient for the purpose of resolving more moderate triggering expected from previous studies. We calculate that an improvement in completeness magnitude from 3.7 to 3.5 could resolve the expected triggering signal in the International Seismic Center (ISC) catalog taken as a whole, but an improvement to $M 2.0$ would be needed to consistently resolve triggering on a regional basis.
\end{abstract}

\section{Introduction}

The past seven years have experienced a surge in great $(M \geq 8)$ earthquakes relative to the preceding four decades (Ammon et al., 2010). Between the $M_{\mathrm{w}} 9.0$ (Global Centroid Moment Tensor) Sumatra-Andaman earthquake of December 2004 and the $M_{\mathrm{w}} 9.0$ Off the Pacific Coast of Tohoku earthquake of March 2011, Earth has averaged 1.7 great earthquakes per year, which is $260 \%$ of the rate of 0.66 per year during the entire seismological record extending back to 1900 (i.e., a rate increase of 160\%). This interval of heightened great earthquake occurrence has prompted many to consider whether the global increase could represent long-range interactions between great earthquakes (Brodsky, 2009; Michael, 2011; Shearer and Stark, 2011); can the occurrence of one great earthquake increase the likelihood of a subsequent quake in a self-exciting process? This certainly seems to be the case for some of the nearby $M \geq 8$ earthquakes. Examples include the 2005 and 2007 Sumatran earthquakes that fol-

*Now at Lamont-Doherty Earth Observatory, Columbia University, Palisades, New York. lowed the 2004 Sumatran-Andaman earthquake and ruptured portions of the plate boundary to the southeast of the first event (Nalbant et al., 2005; Wiseman and Burgmann, 2011), and more recently, the complicated intra-plate rupture to the southwest in 2012 (Meng et al., 2012). The 2006-2007 Kuril doublet is also an example of an $M \geq 8$ earthquake triggered by an earlier event of similar magnitude. These local triggering cases can be interpreted as very large primary or secondary aftershocks of the original quakes. However, even after these local instances are removed, there remains a surfeit of global great earthquakes-1.14 per year, almost double the century-long average rate. Could very large magnitude earthquakes have an extended reach, beyond that for conventionally accepted aftershocks, such that they can trigger earthquake cascades at great distances on 1-10 year timescales?

The peak dynamic strains associated with the surface waves of great $M \geq 8$ earthquakes exceed $10^{-6}$ at global distances, and such strains are commonly observed to trigger small earthquakes (Gomberg and Johnson, 2005; Hill and 
Prejean, 2007; Velasco et al., 2008; van der Elst and Brodsky, 2010; Lei et al., 2011). Indeed, there are reports of triggering of remote activity for many of the recent great events. Examples include triggering in China by the $2003 M_{\mathrm{w}} 8.3$ Tokachi-Oki and $2004 M_{\mathrm{w}} 9.0$ Sumatra-Andaman earthquakes (Lei et al., 2011; Wu et al., 2011), triggering in Alaska and the continental United States by the $2004 M_{\mathrm{w}} 9.0$ Sumatran-Andaman earthquake (West et al., 2005; Rubinstein et al., 2011), triggering in California by the 2010 $M_{\mathrm{w}} 8.8$ Maule-Chile earthquake (Peng et al., 2010), and triggering throughout the United States by the $2011 M_{\mathrm{w}} 9.0$ Off the Pacific Coast of Tohoku earthquake (Rubinstein et al., 2011). Dynamic triggering of remote events is no longer controversial. However, earthquakes larger than about an $M 5.0$ have not yet been observed to be dynamically triggered during passage of great event surface waves (Parsons and Velasco, 2011). Nevertheless, statistical aftershock models predict that sequences of smaller events may occasionally culminate in delayed events that are larger than the initial events (Felzer et al., 2002; Felzer et al., 2004). A good example of this process is the $M_{\mathrm{L}} 5.6$ Little Skull Mountain earthquake, which occurred $280 \mathrm{~km}$ distant and $22.3 \mathrm{~h}$ after the $1992 M_{\mathrm{w}} 7.3$ Landers earthquake, but was preceded by a sequence of small events initiated within the coda of the Landers mainshock (Anderson et al., 1994).

This motivates considering the possibility of a global earthquake cascade, which we test in this study. The model is as follows: large earthquakes radiate powerful surface waves that dynamically trigger small events at global distances. These remotely triggered aftershocks trigger their own aftershocks and so on. Occasionally, secondary aftershocks are larger than the initial mainshock, ramping up the local seismicity rate. This process continues and may eventually culminate in another great earthquake, at which point the global cycle is renewed. Any specific remotely triggered aftershock sequence is highly unlikely to produce an aftershock larger than the initially triggered events, let alone an $M \geq 8$ quake, but if a sufficient number of small, remotely triggered aftershock sequences are initiated globally, the cumulative probability of a few sequences producing big events during a multiyear period may be large.

A remotely triggered rate increase of $160 \%$ may be considered extreme even for the largest of the recent great earthquakes, with the exception of those located in highly susceptible regions. If triggering susceptibility were uniform worldwide, then merely extending uniform aftershock statistics to a global scale would predict an accompanying rise in the rate of smaller earthquakes; that is, there would be many more sequences that culminated with earthquakes of magnitude $M$ 5-7 than those that culminated with an earthquake of $M \geq 8$. Previous studies have not identified a global increase in the number of smaller magnitude earthquakes worldwide (Ammon et al., 2010; Michael, 2011; Shearer and Stark, 2011). We therefore seek to establish a more restricted form of the triggering cascade, in which increases in trigger rates are localized to the sites of some or all recent great earth- quakes. If established, this would indicate that the dynamic triggerability of a fault zone reflects the accumulation of stress on the fault, such that the sites of impending $M \geq 8$ earthquakes are in a state of enhanced susceptibility.

Because transient stresses generated by seismic waves are much smaller than the total strength of a fault or the stress drop in an earthquake (Gomberg et al., 2004), seismic waves must act as the final straw, pushing an already critically stressed fault over the edge to failure. This expectation is guided by laboratory experiments designed to simulate dynamic triggering on stick-slip faults (Beeler and Lockner, 2003; Savage and Marone, 2008) and by numerical studies of theoretical earthquake nucleation models (Gomberg, 2001). These results imply that when and where triggered earthquakes are observed, we may be able to infer the presence of critically stressed faults. It is not known, however, whether the rupture zone as a whole is critically stressed before a great earthquake; that is, whether an earthquake knows in advance how big it is going to get. Susceptibility to dynamic triggering may therefore serve as a probe of the state of stress before (and after) a great earthquake.

Here, we consider the seismic catalog of the past 13 years, using the cascade model to guide the search for distant triggering. If the recent great earthquakes are part of a global self-exciting cascade, the rate of small earthquakes at the site of some or all impending great earthquakes should have increased at the time of some previous large earthquake(s). We therefore search systematically for triggered rate changes localized near the epicenters of subsequent great earthquakes at the time of earlier great earthquakes in the sequence. We look for triggering by all of the $M \geq 8$ earthquakes identified in the Prompt Assessment of Global Earthquakes for Response (PAGER) catalog since 1998, using two global earthquake location catalogs, Preliminary Determination of Epicenters (PDE) and the International Seismological Center (ISC), and one regional catalog, the Japan Meteorological Agency (JMA; see Data and Resources). We measure rate changes using the interevent time ratio method (Felzer and Brodsky, 2005; van der Elst and Brodsky, 2010), and evaluate the significance of any detected rate changes with respect to a Poisson process on a region-by-region basis.

\section{Previous Statistical Studies on Global Earthquake Rate Change}

Some studies have looked at whether recent earthquake rates on a global scale are consistent with a stationary, uniform Poisson process (Michael, 2011; Shearer and Stark, 2011). These two studies examined the statistical distribution of $M \geq 7$ earthquake interevent times during the past century using a global seismicity catalog, and found that the observed clustering of great earthquakes is not significantly non-Poissonian, aside from regional clustering (as expected for proximate aftershocks). This is especially true when we consider the probability of observing strong clustering at some point in a 111-yr earthquake catalog. 
While we cannot demonstrate that the rate of great earthquakes is significantly non-Poissonian, inability to falsify the uniform Poisson hypothesis is not necessarily proof of constant uniform rate. By convention, scientific hypothesis testing is conservative, and we are usually more concerned with minimizing the probability that the null hypothesis is falsely rejected (Type I error) than falsely accepted (Type II error), and it is preferable to use the simplest model possible as the null hypothesis. However, in considerations that have major societal implications, as in the case of global earthquake clustering, it is important to quantitatively address both types of error, that is, to quantitatively assess our confidence that the rate has not increased.

Addressing the potential for Type II error is equivalent to asking: What is the maximum rate change that could still pass the Poissonian hypothesis test at $95 \%$ confidence? That is, what are the confidence bounds on the rate change given the limitations of the catalog? Given $N$ observed earthquakes, the $95 \%$ confidence bound on the Poissonian rate parameter $\lambda$ is just the highest value of $\lambda$ that would produce $N$ or fewer events at least $5 \%$ of the time.

To briefly illustrate this concept, we break down the earthquake record into periods between 1900 and December 2004, and after December 2004 (a prominent change point). This is only an illustration of the concept, and does not constitute a test of the uniform Poissonian hypothesis. We decluster the catalog by removing events within three years and $8^{\circ}$ of any previous event, with the limits chosen specifically to classify the 2005 and 2007 Sumatra and 2007 Kuril earthquakes as aftershocks. The declustered intensity in the first period is $\lambda_{0}=0.56$ events/yr. With nine declustered events in the subsequent seven years, we can be $95 \%$ confident that the rate in the second period is greater than 0.67 and less than 2.24 events/yr. The corresponding rate change $\delta \lambda \equiv \Delta \lambda / \lambda_{0}$ is $19 \%-299 \%$, which covers the naive observation of an apparent rate increase of $160 \%$. The large confidence bounds indicate that the test has very little power to reject the Poisson hypothesis in such a small time window. We cannot statistically rule out a large rate increase, anymore than we can rule out uniformity in rate. Thus, purely statistical treatments of the specific earthquake history are not likely to resolve the question of long-range interactions. More direct examination in the context of a physically motivated framework may provide firmer conclusions.

This paper is organized in this format: First, we introduce the interevent time statistic and define the quantities to be measured. We then describe the spatial and temporal windowing of the catalogs. Before applying the statistic to the real catalog, we calculate the expected rate change at each site, given previously established scaling laws for remote triggering, so that we can interpret the incidence of nondetections. We then proceed to measure actual triggered rate changes near the epicenters of great earthquakes, both before and after they occur, calculating significance with respect to a homogeneous Poisson process. We identify no precursory triggering before impending great earthquakes that could explain the global rate increase. The simple, homogeneous Poisson model is not a particularly good representation of the true temporal clustering of earthquakes, so we also develop a nonstationary reference model that accounts for mainshock-aftershock clustering. There is a possible signal showing short-term precursory triggering with respect to the nonstationary reference, but the evidence is inconclusive. Finally, we use the information gathered at each target site to assess how much improvement would need to be made to the global seismic network in order to detect remote triggering at the sites of major subduction zone earthquakes on a consistent basis.

\section{Method}

\section{Interevent Time Ratios}

To capture rate changes in the impending rupture zones of great earthquakes, we take the sample mean of the interevent time ratio $r$, calculated in regional spatial bins, defined by

$$
r=\frac{t_{2}}{t_{1}+t_{2}},
$$

where $t_{1}$ and $t_{2}$ are the intervals to the first local earthquake before $\left(t_{1}\right)$ and after $\left(t_{2}\right)$ some reference time-in this case, the time of a distant great earthquake. If the regional earthquake times are uncorrelated with the time of the distant great earthquake, $r$ is uniformly distributed between $(0,1)$. If the timing of regional earthquakes is advanced by the occurrence of the distant great earthquake (i.e., if there is a remotely triggered earthquake or one of its aftershocks in the local bin), $t_{2}$ will be on average smaller than $t_{1}$, and the distribution of $r$ will be shifted toward smaller values.

Each great earthquake target zone is divided into a uniform grid, and $r$-values are calculated from pairs of earthquakes within each grid square. The target zones are defined by the first 10 days of aftershocks of each great earthquake, extending no further than $1^{\circ}$ from the mainshock epicenter. The spatial constraint focuses attention on the plausible nucleation regions for the great events. The grid spacing within this region is optimized to give the maximum number of unique pairs of earthquakes in a region. (Only one value of $r$ can be calculated for each grid square, so the number of unique pairs increases with the fineness of the grid, up to the point where bins begin to lack earthquake pairs straddling the trigger events.) This optimization is done for each trigger-region pair.

The population of $r$-values for each target zone is averaged, giving a mean $r$ (denoted $\bar{r}$ ), and this mean is compared with the expected mean for a reference model (e.g., uniform Poisson process, Appendix A). The triggering detection confidence is defined as $c=1-p_{r}$, where $p_{r}$ is the significance, that is, the probability that a random process would produce a mean as small or smaller than the observed $\bar{r}$, by chance. It should be noted that in using this one-sided definition, the triggering confidence equals $50 \%$ for zero rate 
Table 1

Symbols Used in This Study

\begin{tabular}{cl}
\hline$r$ & Interevent time ratio \\
$\bar{r}$ & Sample mean of $r$ \\
$\bar{r}_{\text {corr }}$ & Model adjusted $\bar{r}$ \\
$\lambda$ & Earthquake rate \\
$\delta \lambda$ & Rate change \\
$\delta \lambda_{\mathrm{xx}}$ & Rate change, xx\% confidence bound \\
$\delta \lambda_{\mathrm{exp}}$ & Expected rate change \\
$m$ & Sample size \\
$p_{r}$ & Statistical significance of $\bar{r}$ \\
$p_{50}$ & Significance of proportion of $p_{r}<0.5$ \\
$p_{90}$ & Significance of proportion of $p_{r} \leq 0.1$ \\
\hline
\end{tabular}

change, and a triggering confidence near zero means that an apparent rate decrease is present. However, this definition restricts us from assigning negative confidence to values of $\bar{r}<0.5$.

We also calculate a fractional rate change $\delta \lambda$ consistent with the observed $\bar{r}$, assuming that the earthquake rate is constant and Poissonian, but with a step-change in rate at the time of the trigger (Appendix A). The fractional rate change $\delta \lambda$ is defined as the difference in rates, normalized by the initial rate. We also compute the upper and lower $95 \%$ confidence bounds on $\delta \lambda$, which depend on both $\bar{r}$ and the sample size (Appendix A). The upper and lower bounds can be thought of as the highest and lowest rate changes that could still pass a Poissonian hypothesis test. These three valueslower bound, best estimate, and upper bound-are denoted $\delta \lambda_{05}, \delta \lambda_{50}$, and $\delta \lambda_{95}$, respectively. (See Table 1 for a list of symbols used in this study.)

\section{Triggers and Target Regions and Time Windows}

The trigger and target regions consist of all $M \geq 8$ earthquakes since the $M_{\mathrm{w}} 8.1$ Balleny Islands earthquake of 1998 up to the $2011 M_{\mathrm{w}}$ 9.0 Off the Pacific Coast of Tohoku earthquake (Fig. 1 and Table 2). This start date is chosen because we consider the JMA catalog to be of consistent quality only after 1997. This end date does not include the most recent 2012 Sumatran earthquakes, which occurred after finalization of this study. (We note, however, that preliminary calculations show no distant triggering by the 2012 Sumatran earthquake at the epicenters of the previous great earthquakes in this study, and only the nearby 2004 and 2005 Sumatran earthquakes triggered seismicity at this target site.) Earthquake magnitudes are taken from the PAGER catalog, and all correspond to $M_{\mathrm{w}}$ values from centroid moment tensor inversions.

The window for measuring triggering relative to each great event is limited so that triggering windows do not overlap with other trigger times (windows themselves may overlap but cannot contain more than a single $M_{\mathrm{w}} \geq 8$ trigger event). Allowing more than one trigger in each window could lead to double counting and would allow the impending regional

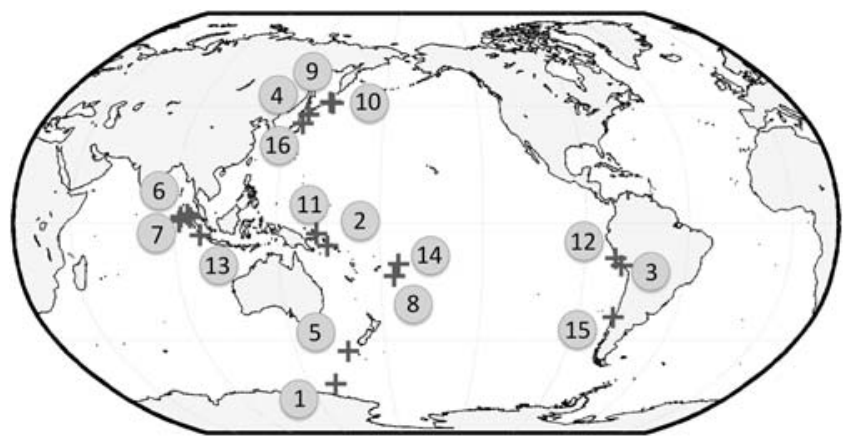

Figure 1. Locations of $M_{\mathrm{w}} \geq 8$ great earthquakes used in this study. Crosses mark locations; numbers give the order of occurrence (Table 2).

quake itself or its aftershocks to be considered a remotely triggered event. Double counting, or counting the $M_{\mathrm{w}} \geq 8$ earthquakes themselves (which were used to formulate the hypothesis), would invalidate the statistical significance calculations. Each time window is also constrained to be symmetrical around the great event trigger time, as the interevent time ratio $r$ would be biased if the waiting times $t_{1}$ and $t_{2}$ were limited to different ranges.

The choice of target time window and target region do not strongly influence the conclusions of this paper. Using smaller time windows has the effect of diminishing the sample size and reducing statistical robustness, but larger time windows have the effect of averaging out any triggered rate increase over the duration of the window. Using a constant, smaller maximum time window of \pm 59 days, for example, changes the joint statistics by $1 \%-2 \%$ but does not alter any of the conclusions of this study. We choose to use variablelength windows because it maximizes the number of samples overall.

The requirement of nonoverlapping windows is a problem for the 2004 Macquarie Islands and 2004 SumatraAndaman earthquakes, which are separated by only 2.4 days. This could be taken as a perfect example of one great earthquake being triggered on the heels of a $77^{\circ}$ distant earthquake. However, no significant precursory triggering of smaller events due to the Macquarie earthquake is observed in the rupture region of the impending Sumatra quake (there is only one earthquake near Sumatra in the interval between them reported in the ISC catalog). Rather than impose a 2.4-day time limit on both earthquakes, we choose to expand the triggering window to the limits imposed by the previous and next events in the sequence ( $\sim 95$ days rather than 2.4). This means that any triggering by the Sumatra-Andaman quake may be attributed to the Macquarie quake and vice versa. These two triggers are considered as a single event when calculating the joint statistical significance of measured rate changes.

Triggering susceptibility is measured using three target catalogs. We first examine the PDE and ISC global earthquake catalogs. The PDE is a rapidly published compilation 
Table 2

List of Great Earthquakes

\begin{tabular}{rclccc}
\hline & Date (yyyy/mm/dd) & \multicolumn{1}{c}{ Name } & $M_{\mathrm{w}}$ & $M_{\mathrm{C}}$ (PDE/ISC/JMA) & $T$ (days) * \\
\hline 1 & $1998 / 03 / 25$ & Balleny Island & 8.1 & $5.6 / 4.1 /-$ & 83 \\
2 & $2000 / 11 / 16$ & New Ireland & 8.0 & $4.2 / 3.6 /-$ & 220 \\
3 & $2001 / 06 / 23$ & Peru & 8.4 & $4.6 / 3.6 /-$ & 220 \\
4 & $2003 / 09 / 25$ & Tokachi-Oki & 8.3 & $4.0 / 3.5 / 1.3$ & 455 \\
5 & $2004 / 12 / 23$ & Macquarie Island & 8.1 & $5.2 / 5.1 /-$ & 95 \\
6 & $2004 / 12 / 26$ & Sumatra-Andaman & 9.0 & $4.3 / 3.8 /-$ & 93 \\
7 & $2005 / 03 / 28$ & Sumatra-Nias & 8.6 & $5.5 / 3.7 /-$ & 93 \\
8 & $2006 / 03 / 05$ & Tonga & 8.0 & $4.4 / 3.8 /-$ & 196 \\
9 & $2006 / 11 / 15$ & Kuril Island & 8.3 & $4.6 / 3.5 /-$ & 59 \\
10 & $2007 / 11 / 13$ & Kuril Island & 8.1 & $4.6 / 3.6 /-$ & 59 \\
11 & $2007 / 01 / 04$ & Solomon Islands & 8.1 & $4.3 / 3.6 /-$ & 78 \\
12 & $2007 / 07 / 15$ & Central Peru & 8.0 & $4.1 / 3.5 /-$ & 27 \\
13 & $2007 / 09 / 12$ & South Sumatra & 8.5 & $4.4 / 3.6 /-$ & 27 \\
14 & $2009 / 09 / 29$ & Samoa & 8.0 & $5.0 / 3.7 /-$ & 151 \\
15 & $2010 / 02 / 27$ & Chile & 8.8 & $4.4 / 3.7 /-$ & 151 \\
16 & $2011 / 03 / 11$ & Tohoku-Oki & 9.0 & $4.2 / 3.5 / 1.3$ & 92 \\
\hline
\end{tabular}

*Maximum allowed $t_{1}$ or $t_{2}$ in equation 1 based on the timing of other $M_{\mathrm{w}} \geq 8$ earthquakes. The total trigger time window is $2 T$, centered on each great event.

of many reporting networks worldwide. In the bestinstrumented regions, it has a completeness level of $M_{C}=$ 4.1 and is complete up to the present. The completeness level varies widely by region, however, and is as high as 5.6 in this study. We determine the completeness magnitude for each target region as the magnitude above which a linear fit can explain at least $90 \%$ of the variance in log-cumulativenumber versus magnitude (Wiemer and Wyss, 2000). The regional magnitude of completeness is calculated over the entire rupture area of the target earthquake. We make a slight alteration to the method of Wiemer and Wyss (2000), computing the goodness-of-fit statistic only for magnitude bins that contain at least one earthquake. This prevents the large mainshocks, which often introduce a plateau into the cumulative number distribution, from dominating the misfit. The 90\% completeness magnitudes for each catalog are given in the list of triggers and targets (Table 2).

The goodness-of-fit test of Wiemer and Wyss (2000) is known to give lower estimates of the magnitude of completeness compared with other methods (Mignan and Woessner, 2012). A threshold goodness-of-fit value of $95 \%$ is typically sought when mapping spatial variations in seismicity parameters, and the $90 \%$ value used here is considered somewhat of a compromise. For our purposes, however, a slight underestimate of the magnitude of completeness is acceptable, as there is a tradeoff between catalog completeness confidence and the remaining sample size. Because the interevent time statistic uses normalized rates, it should be robust against minor catalog incompleteness, as long as the completeness level in the target region does not change systematically at the time of the distant trigger.

The ISC catalog is the authoritative final catalog combining the best data worldwide. It has a somewhat lower completeness threshold (minimum $M_{C}=3.5$, regionally up to $M_{C}=5.1$ ), but extends only through June 2010 at this time.
The PDE catalog is therefore the only source of regional target data for the 2011 Tohoku-Oki trigger earthquake. Where the PDE catalog does overlap with the ISC catalog, it provides a qualitative check on the effect of catalog uncertainties on the measured triggering significance, although we consider the ISC catalog to be authoritative. The ISC includes unreviewed hypocenter data below magnitude 3.5 , the quality of which is very nonuniform in time. We therefore restrict the ISC catalog to prime quality events above $M$ 3.5. Finally, the regional JMA catalog is used for target regions near Japan. This catalog has a much lower $90 \%$ completeness threshold $\left(M_{C}=1.3\right)$ in the Tokachi-Oki and Tohoku-Oki target regions.

\section{Binomial and Joint Significance Tests}

We compute the significance of $\bar{r}$ for each of the 256 individual trigger-target pairs (16 triggers at 16 target regions), with respect to the reference model (e.g., uniform Poisson process) and report triggered rate change detection successes as instances with greater than $90 \%$ triggering confidence. This represents a relatively low significance threshold, designed to capture relatively low rate changes in small samples, and it requires that we always consider the number of detection thresholds expected by chance when interpreting the results for any subgroup of the data. We, therefore, also compute the joint significance for four subgroups of the data, as follows: (1) the subset of all 120 trigger-target pairs that come any time before the regional earthquakes, reflecting long-term precursory triggering (referred to as All Precursory); (2) the subset of 15 pairs from the precursory group that come most recently in the sequence before each regional earthquake, reflecting short-term precursory triggering (First Preceding); (3) the subset of all 120 pairs that come any time after the regional earthquakes (All Subsequent); and 
(4) the subset of 15 pairs from the All Subsequent group that fall next in the sequence after each regional earthquake (First Subsequent). The two precursory groups tell us about any potential earthquake cascade or precursory triggering, and the two subsequent groups tell us about changes in triggerability in response to damage induced by a large regional quake. The labels in parentheses will be used in the tables throughout.

We compute the combined significance in these subgroups, using the same method as in the case-by-case basis, that is, as the probability of obtaining a smaller $\bar{r}$ by chance for the entire population (the $p$-value), denoted $p_{r}$. However, computing $p_{r}$ in this way weights the calculation toward the sites with the most earthquakes, and this may dampen the triggering signal from more sparsely covered regions. For this reason, we also compute the significance of the number of detection success within each group, treating each measurement as a Bernoulli trial with probability of success $1-c_{\mathrm{th}}$, where $c_{\mathrm{th}}$ is the detection confidence threshold. The triggering signal could take several forms: occasional high confidence triggering at a few regions, or a systematic small bias toward slightly positive rate changes. To capture both of these cases, the binomial test is computed for thresholds of $90 \%$ and $50 \%$, with $p$-vaues denoted $p_{90}$ and $p_{50}$. The results of these tests are considered statistically significant if the $p$-values fall below 0.05 .

In this paper, we follow the convention that case-by-case triggering measurements will be reported in terms of triggering confidence, and all joint statistics derived from multiple confidence measurements will be reported in terms of significance level $p$.

Expected Rate Changes from Surface-Wave Strain Amplitudes

In order to interpret the incidence of nondetections, we also calculate an expected rate change based on previous regional observations. Van der Elst and Brodsky (2010) calibrated a relationship between the amplitude of triggering waves and the triggered rate change using the interevent time ratio $r$ in California. Fitting a power law to the triggered rate change versus peak surface-wave strain $\varepsilon$ in that study gives an expected rate change of

$$
\delta \lambda_{\exp }=82 \times \varepsilon^{0.43} .
$$

An identical analysis of Japan seismicity found that Japan is approximately three times less triggerable than California, which gives an indication of the variability we should expect in triggering susceptibility worldwide. The triggering sites in this study are also potentially much deeper in this study than in the one used to calibrate equation (2). We therefore treat equation (2) only as a rough estimate of expected rate change, and likely an upper bound.

Van der Elst and Brodsky (2010) estimated peak surface-wave strain using the empirical surface-wave magnitude equation, which relates wave amplitude to earthquake magnitude and distance. For the large magnitude earthquakes we are considering here, the surface-wave magnitude is saturated and the empirical scaling is inadequate. Instead, we measure peak velocities directly at nearby seismic stations (see Data and Resources). Dynamic strain amplitude is estimated as

$$
\varepsilon=\frac{v_{\max }}{c_{S}},
$$

where $c_{S}=3.5 \mathrm{~km} / \mathrm{s}$ is the surface-wave phase velocity, and $v_{\max }$ is the peak vertical velocity measured on a broadband sensor. Seismic stations are selected within $6-10^{\circ}$ of the target earthquake epicenter, depending on the density of stations, and the instrument response is removed over a passband of 1-50 s. Amplitude at the target earthquake epicenter is extrapolated from the measured values using the distance decay exponent from the surface-wave magnitude equation $M_{S}=\log A+1.66 \log \Delta+2$ (Lay and Wallace, 1995). Where multiple records exist, the amplitudes are distance corrected and averaged. In the 14 out of 256 cases where no amplitude measurements exist within $10^{\circ}$ of the epicenter, the trigger amplitude is estimated as the mean of the distancecorrected amplitudes measured at all the other target sites.

The measured peak ground velocities agree well with the empirical surface-wave amplitude equation multiplied by $2 \pi / T$ (period $T=20 \mathrm{~s}$ ) to get velocity but with somewhat reduced dependence on magnitude, as expected for this magnitude range. There is also antipodal focusing of the surface waves beyond the $150^{\circ}$ distance that is not captured by the empirical surface-wave equation.

Using equations 2 and 3 to transform measured peak velocity into expected rate change, we determine a mean expected rate change $\delta \lambda_{\text {exp }}$ over all trigger-target pairs on the order of $11 \%$ (Fig. 2). This expectation is for rate change averaged over a large area, and triggered rate change may be much higher locally. Indeed, it must be higher locally if triggering is to account for the observed increase in the rate of large earthquakes.

Such small rate changes require large sample sizes to establish high significance (Table 3; Appendix A). For example, a sample size of $m=11$ is required to establish a $100 \%$ rate change (doubling) at $90 \%$ confidence. A sample size $m=543$ would be required to establish a rate increase of $10 \%$. To establish a $1 \%$ rate change at $90 \%$ confidence would require $m=49,765$. Given the limited completeness of the global seismicity catalog, it may be possible to resolve rate changes of $10 \%-100 \%$ on a regional basis, but smaller rate changes $(<10 \%)$ will likely go unnoticed.

\section{Results}

\section{Example Target Location}

As an example, the target region for the Tohoku-Oki earthquake is shown in Figure 3, using the JMA catalog, for all 16 of the potential trigger earthquakes (Table 2), with 


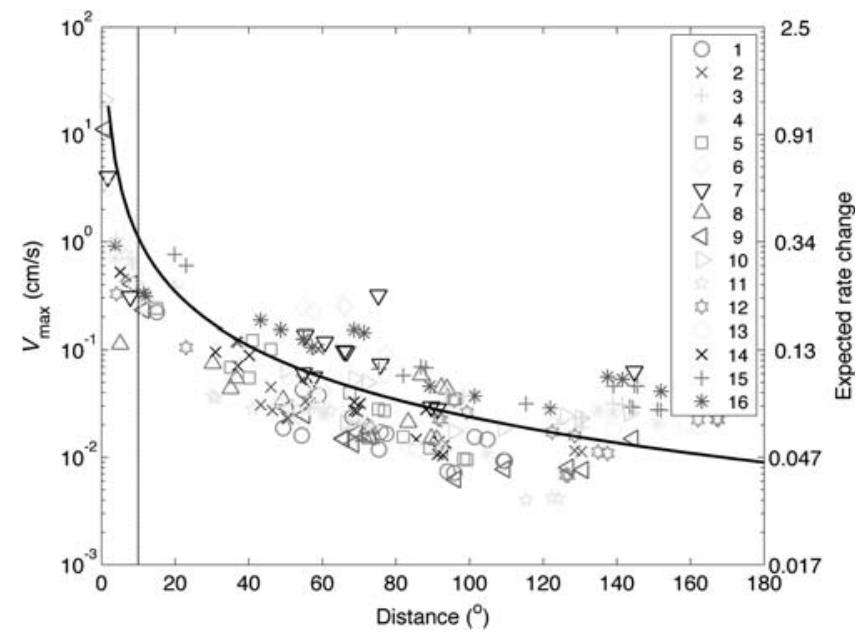

Figure 2. Peak surface-wave velocity and expected rate change $\delta \lambda_{\exp }$ (Eq. 2) for each $M \geq 8$ earthquake, at each target site. The legend gives the earthquake number from Table 2 (e.g., 1, 1998 Balleny Islands; 6, 2004 Sumatra-Andaman; 16, 2011 Tohoku-Oki). The black curve shows the empirical surface-wave magnitude equation, multiplied by $2 \pi / T$ (period $T=20 \mathrm{~s}$ ) to obtain velocity, for an $M_{\mathrm{S}} 8.2$ earthquake (the median trigger magnitude in this study). The dashed line shows the $10^{\circ}$ limit used to define the far field. The increase in amplitude beyond $150^{\circ}$ is due to antipodal focusing.

triggers five and six combined. This is the best-instrumented target region out of all 16 targets $\left(M_{C}=1.3\right)$, and therefore the density of earthquake pairs is very high. Most other sites, using the global earthquake catalogs, are much more poorly sampled.

One trigger beyond $10^{\circ}$ distance produces a mildly significant triggering signal (93\% confidence) within the target region: the 2003 Peru earthquake, although it is not visually apparent (Fig. 3, Trigger 3). The nearby (3.7 ) 2004 Tokachi-Oki earthquake (Fig. 3, Trigger 4) more clearly triggered earthquakes within the epicentral region of the 2011 Tohoku-Oki quake, with $p$-value $p_{r}<10^{-16}$. Examining the effect of the Tohoku-Oki mainshock on its own epicentral region, we see that the $M 7$ foreshock sequence, initiated two days prior, shows up as a small patch of apparent triggered rate decrease (blue dots, Fig. 3, trigger 16), within a robust conventional aftershock sequence that fills in the frame to the northeast and southwest.

\section{Global ISC Catalog-Uniform Poisson Reference Model}

We now apply the interevent time statistic to the global ISC catalog. We measure triggering significance with respect to a stationary, uniform Poisson reference model. As mentioned in the Introduction, this is an imperfect model, and it may spuriously map any non-Poissonian clustering into the triggering signal. This is problematic because we know that a Poisson process is a very poor approximation of the aftershock sequences of great earthquakes. We will subsequently correct for this by introducing a nonstationary reference model
Table 3

Sample Size Required to Detect a Triggered Rate Change

\begin{tabular}{ccc}
\hline $\begin{array}{c}\text { Rate } \\
\text { Change } \delta \lambda[\%]\end{array}$ & Expected $\bar{r}$ & $\begin{array}{c}\text { Required Sample Size } m \\
\text { for } 90 \%(95 \%) \\
\text { Confidence }\end{array}$ \\
\hline 1 & 0.498 & $49,765(81,980)$ \\
5 & 0.492 & $2071(3411)$ \\
10 & 0.484 & $543(895)$ \\
67 & 0.415 & $20(32)$ \\
100 & 0.386 & $11(18)$ \\
160 & 0.345 & $6(10)$ \\
\hline
\end{tabular}

that accounts for aftershocks. However, we first present the data using the over-simplified stationary reference model in order to clearly demonstrate which of our conclusions are model dependent. The equivalent analyses for the PDE and JMA catalogs, with respect to the uniform Poisson reference model, are included in Appendix B.

Applying the interevent time test to all sets of triggers and target regions gives a matrix of triggering detections (Fig. 4). The triggers are sorted by time of occurrence along the $y$-axis, and sorted as target regions along the $x$-axis, such that boxes above the diagonal represent periods after the time of the great earthquake in that region, and boxes below the diagonal represent periods before the occurrence of the regional great earthquake. Trigger-target pairs that are less than $10^{\circ}$ distant are marked with red squares, and are not included in the joint remote triggering statistics.

The diagonal of the triggering matrix shows the effect of a trigger earthquake upon its own rupture area (Fig. 4). Triggering of conventional aftershocks along this diagonal is detected with very high confidence $\left(p_{r}<10^{-16}\right)$. Beyond $10^{\circ}$ distance, however, systematic triggering is not evident, with $p_{r}=0.38$ (Table 4).

The lower right triangle of the triggering matrix (Fig. 4) is the region to examine for possible triggering precursory to great earthquakes. We do not detect systematic triggering for separations greater than $10^{\circ}$, with a joint triggering significance over the whole precursory population of $p_{r}=0.46$ (Table 4).

The boxes immediately to the right of the center diagonal are the places to look for triggering by the most recent great earthquake in the sequence, that is, the First Preceding subgroup (Fig. 4). In a model where each earthquake advances the next earthquake in the sequence in a binary manner, this would be the place to expect a triggering signal. The signal for this subgroup is the strongest out of any subset, although not quite significant $\left(p_{r}=0.09\right)$. The signal is dominated by triggering by the 2010 Chile earthquake at the site of the impending 2011 Tohoku earthquake, although we note that this one detection success is not significant given the number of detection attempts $\left(p_{90}=0.61\right)$.

The upper left triangle of the matrix (Fig. 4) is the region to examine for possible triggering in regions after a 

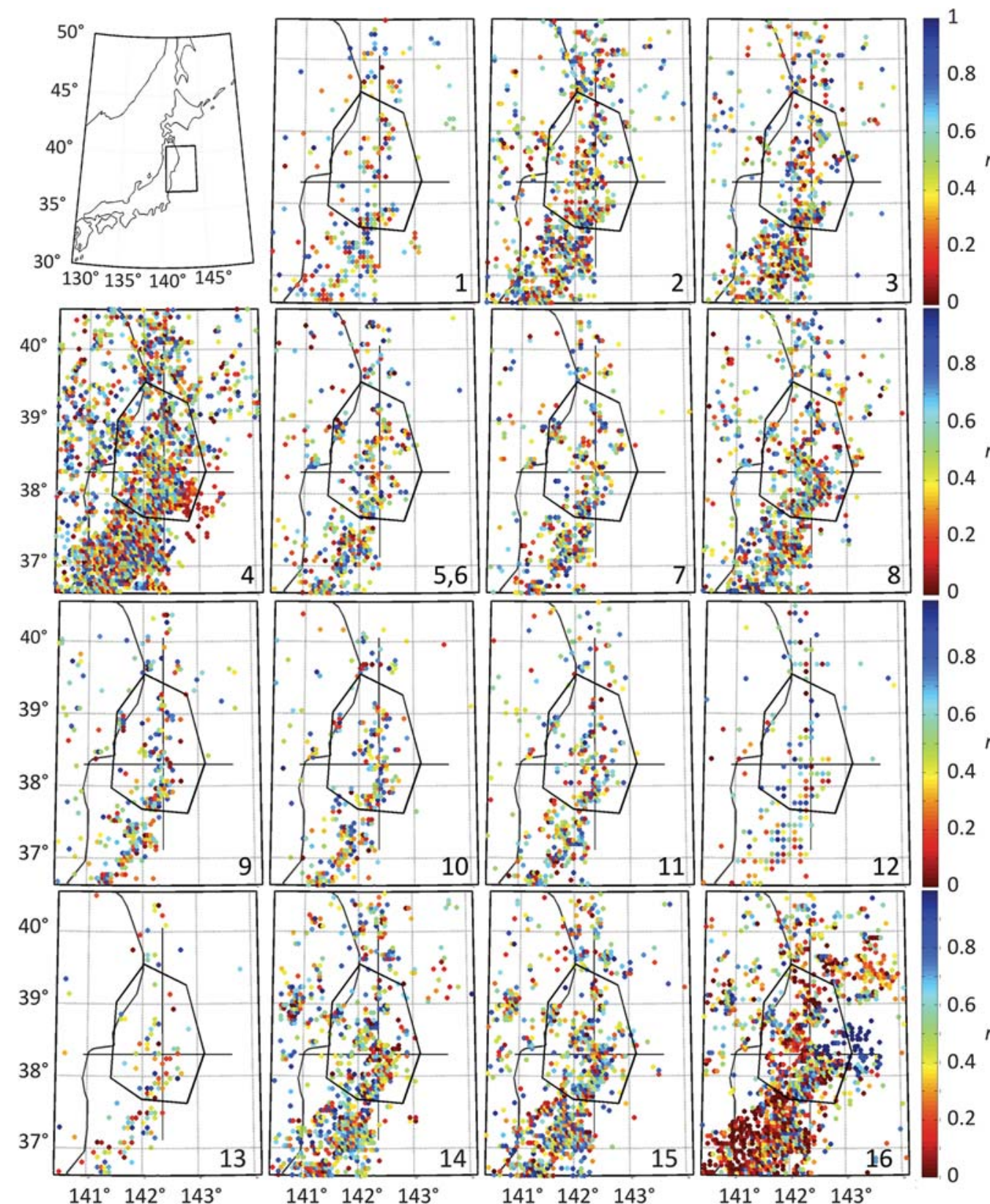

Figure 3. The Tohoku-Oki target region for all 16 triggers (numbered in the bottom right of each panel). The top left panel is the context map. The crosshairs mark the epicenter of the $2011 M_{\mathrm{w}} 9.0$ Off the Pacific Coast of Tohoku-Oki quake, and the polygon outlines the first 10 days of Tohoku-Oki aftershock activity within $\sim 1^{\circ}$ of the epicenter. The region is gridded on a fine scale (optimized for each trigger), and each colored dot represents one pair of earthquakes within that grid-one before and one after the trigger time. The color corresponds to the $r$ value (equation 1); warmer colors indicate larger triggered rate change.

great earthquake. This subgroup also does not show strong triggering, with a joint triggering significance of $p_{r}=0.37$ (Table 4).

The ISC catalog does not show significant triggering, for any subset, against a Poissonian reference. However, we know that the actual regional time series are not Poissonian, but rather follow mainshock-aftershock clustering statistics. In the next section we examine the effect that mainshock-aftershock clustering may have on the interevent time statistics, and develop a more sophisticated reference model.

\section{Methods Revisited}

Limitations of the Uniform Poisson Reference Model

In the previous section, the significance $p_{r}$ was calculated with respect to a uniform Poisson process (Appendix A). However, the regional catalogs are very non-Poissonian due to local mainshock-aftershock clustering. This is a particularly serious problem for this study, because the selection of target time windows is conditioned on the recent occurrence of large earthquakes - and hence their aftershocks - at the beginning of the window. 


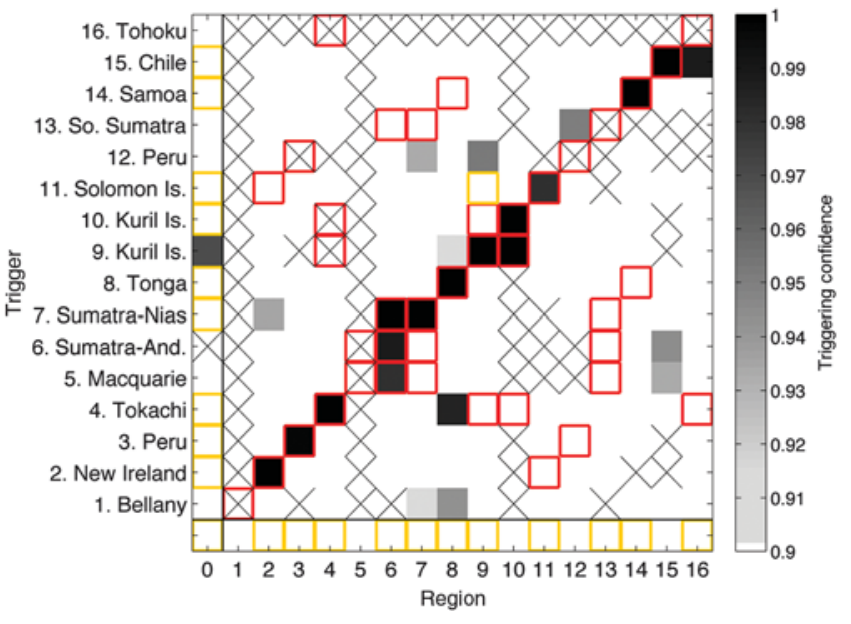

Figure 4. Triggering matrix for the ISC catalog, with significance measured with respect to a stationary, uniform Poisson process. The triggering matrix shows regions with rate increases above $90 \%$ confidence (grayscale). Triggers are sorted by date along the $y$ axis, and sorted as target regions on the $x$-axis. Yellow squares show where a rate increase of $67 \%$ could theoretically be detected or excluded. Red squares mark events within $10^{\circ}$ of each other. The far left column shows the combined triggering confidence for each trigger over all target regions (excluding those in red squares), and the bottom row shows the combined confidence for each region over all triggers (excluding red squares). The bottom left square gives the triggering confidence for the entire catalog. Crosses indicate no data for that trigger-target pair.

Figure 5 shows the effect of taking a random sample of interevent time ratios from within an ongoing aftershock sequence, and calculating significance with respect to a uniform Poisson reference model. Even though aftershock rates are decaying, which intuition suggests might produce a spurious rate decrease in the apparent triggering metric, the $r$ statistic actually indicates a spurious rate increase. This is because times are drawn from a finite time window, and not every spatial bin will contain an earthquake both before and after the trigger time. The presence of aftershocks near the beginning of the pre-trigger time window makes it much more likely that a bin will fail to be included on account of a too-large $t_{2}$ than a too-large $t_{1}$. The remaining $t_{2}$ 's tend to be more uniformly distributed than the $t_{1}$ 's, which are con-

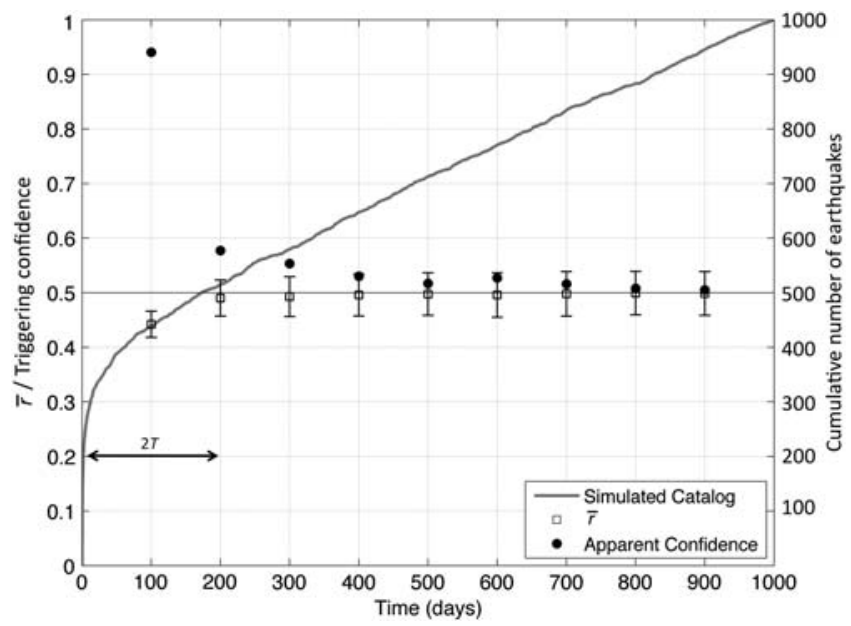

Figure 5. Illustration of the effect of early aftershocks on apparent triggering as measured by the interevent time statistic $r$. The gray line is the cumulative number of earthquakes in a simulated catalog containing 50\% background events and 50\% aftershocks decaying according to Omori's law. The squares show measurements of $\bar{r}$ for hypothetical distant earthquakes occurring at 100 -day intervals. The simulated target window is $2 T=200$ days long. The simulation contains no long-range triggering effects, therefore the skew downward of $\bar{r}$ near the beginning of the aftershock sequence and associated skew upwards of the apparent triggering confidence (circles) are effects of the aftershock sequence itself on the statistic.

centrated toward the beginning of the time window, and therefore toward larger values. In this way, the finite window produces a positive bias in the rate-change calculation.

In order to accurately measure the effect of remote triggering at the time of the trigger, we must compute the significance of the measured $\bar{r}$ with respect to a modeled distribution that accounts for local clustering at the beginning of the time window.

\section{Significance with Respect to a Nonstationary Reference Model}

The appropriate distribution of the expected value of $\bar{r}$ is modeled using a simulated catalog that reproduces the heterogeneities in rate due to local mainshock-aftershock sequences. We call this model the nonstationary or Omori

Table 4

Statistical Significance: ISC Catalog_Poisson Reference

\begin{tabular}{lcccccc}
\hline & $\bar{r}$ & $m^{*}$ & $p_{r}$ & $p_{50}$ & $p_{90}$ & $\delta \lambda_{50}\left(\delta \lambda_{05,95}\right)[\%]$ \\
\hline All & 0.390 & 774 & $<\mathbf{1 0}^{-\mathbf{1 6}}$ & 0.20 & $\mathbf{0 . 0 4}$ & $\mathbf{9 6}(77,117)$ \\
All Distant & 0.496 & 468 & 0.38 & 0.47 & 0.87 & $3(-10,17)$ \\
All Precursory & 0.498 & 218 & 0.46 & 0.59 & 0.85 & $1(-16,23)$ \\
All Subsequent & 0.494 & 250 & 0.37 & 0.40 & 0.77 & $4(-13,24)$ \\
1st Preceding & 0.422 & 25 & 0.09 & 0.75 & 0.61 & $60(-10,181)$ \\
1st Subsequent & 0.482 & 41 & 0.35 & 0.50 & 0.23 & $11(-28,73)$
\end{tabular}

* Sample size $m$ is the combined total number of local earthquake pairs $\left(t_{1}, t_{2}\right)$ in each subgroup, which can be greater than the number of great earthquake triggertarget pairs in the subgroup. 
reference model. The model catalog contains two seismicity components: (1) spontaneous background earthquakes with a statistically constant rate $\mu$, and (2) aftershocks of large $(M \geq 8)$ regional and moderate local earthquakes that follow the modified Omori's law, resulting in a combined model rate $\lambda(t)$ defined by

$$
\lambda(t)=\mu+\sum_{i=1}^{n} k_{i}\left(t-t_{i}+c\right)^{-p} u\left(t-t_{i}\right) .
$$

Here, $k_{i}$ are the productivity terms for each of the $n$ aftershock sequences, $c$ and $p$ are constants governing the time decay of aftershocks, and the unit step function $u\left(t-t_{i}\right)$ is zero if $t<t_{i}$ and one otherwise. The rate $\lambda$ at some time $t$ is hence the sum of the constant background rate and all the aftershock rates from mainshocks with times $t_{i}<t$.

Aftershock rates $k_{i}$ are calculated for local mainshocks with a significant effect on the time series. Significant local mainshocks are identified as the minimum population of large local events sufficient to capture the observed cumulative time series within the range of the modeled time series (e.g., Fig. 6). Local mainshocks are modeled only if their inclusion is required to achieve overlap between the modeled and observed cumulative time series and not if they merely improve the fit by some statistical measure. This captures large nonuniformities in rate without over-fitting the time series, which could mask the signal of remote triggering. The number of significant local mainshocks ranges from $n=$ 0 to 5 over the various target regions (Appendix C). The smallest local mainshock magnitude is $M 5$, and the mean magnitude is $M$ 6.4. The model rate also includes aftershocks of the local target $M_{\mathrm{w}} \geq 8$ earthquake, as well as any other regional $M_{\mathrm{w}} \geq 8$ earthquakes within $10^{\circ}$.

To verify that we are not removing the signal we are hoping to measure, we also have run tests in which we (1) model only the major $M \geq 8$ quakes with no local mainshocks, (2) model only the subset of local mainshocks that precedes each potential trigger, and (3) model several more or several fewer local mainshocks. None of these alternative tests results in a qualitative change in the joint statistics for any region, trigger, or subset of the triggering matrix, and we do not include these tests in detail here.

The optimal $\mu, c, p$, and $k_{i}$ (equation 4) are found using the maximum likelihood objective function of Ogata, 1992, and the Matlab constrained optimization routine fmincon, to enforce positivity of the parameters. The productivity terms $k_{i}$ are not assumed to depend on magnitude through a productivity law as in traditional ETAS models (Ogata, 1992). Instead, each $k_{i}$ is treated as a free parameter. This is superior to an a priori productivity law because the relatively small epicentral target windows tend to crop aftershock sequences in space, disrupting the usual magnitude-productivity scaling. Our method simply attributes the triggered rate increase within the target region to the first event of the sequence (mainshock or aftershock) that happens to fall within the target region. This method produces robust inversions as long as the

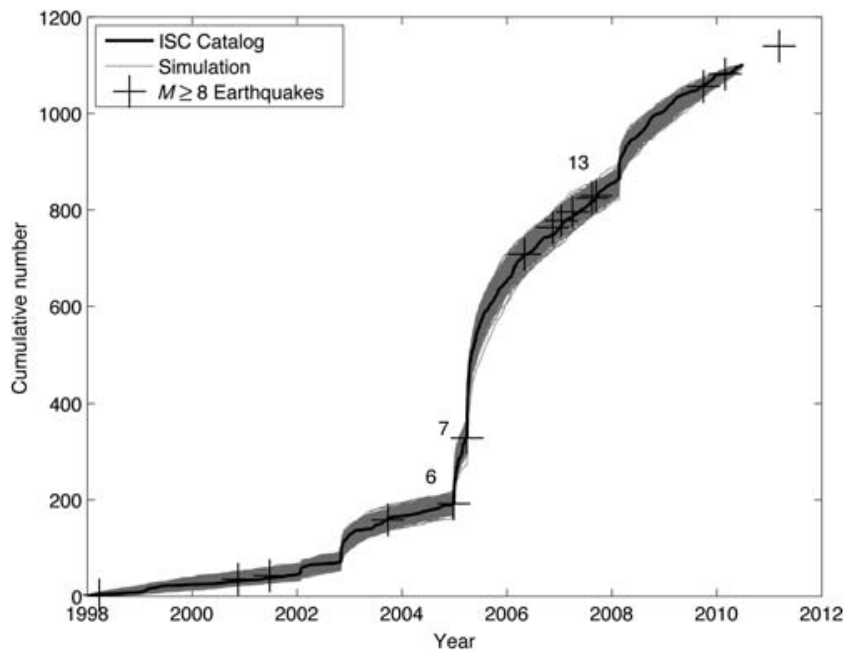

Figure 6. Cumulative observed seismicity obtained from the International Seismic Center (ISC) catalog in the 2004 Sumatra target region (black line) along with 1000 simulated time series (gray lines) generated from the nonstationary Omori model (equation 4). The times of all the $M_{\mathrm{w}} \geq 8$ potential triggers are shown as large crosses. The ISC catalog extends only to June 2010. The local $2004 M_{\mathrm{w}} 9$ Sumatra-Andaman earthquake and the nearby 2005 Sumatra-Nias and 2007 southern Sumatra earthquakes are labeled by trigger number (Table 2). Aftershocks from these three great triggers, as well as three significant local earthquakes-evident as small jumps in seismicity rate, are included in the modeled local seismicity rate.

number of $k_{i}$ remains small. The optimal parameters for each earthquake catalog, as well as the proportion of aftershocks in each target region, are reported in Appendix C.

As an example, Figure 6 shows the observed and simulated catalogs for the epicentral region of the 2004 SumatraAndaman earthquake, which includes the effects of three regional $M_{\mathrm{w}} \geq 8$ trigger earthquakes, as well as three moderate local mainshocks.

To compute significance of the observed $\bar{r}$, we generate 100,000 random catalogs based on the model rate, and then draw simulated samples of interevent time ratios $r$ using the same time windows and sample sizes as present in the real catalog. This leads to a model distribution of values for the expected mean, denoted $\bar{r}_{\text {sim }}$. Significance of the real $\bar{r}$ is determined as the proportion of modeled $\bar{r}_{\text {sim }}$ that are smaller than or equal to $\bar{r}$, that is, $p_{r}=F_{\text {sim }}(\bar{r})$ (Fig. 7). When computing the significance of local triggering for the regional $M_{\mathrm{w}} \geq 8$ event itself, or for other nearby great earthquakes that contribute to the model rate, we remove these particular $k_{i}$ from the modeled seismicity rate.

We also estimate a triggered rate change $\delta \lambda$ based on the measured $\bar{r}$. However, we want to avoid mapping the contribution from catalog nonuniformity into the rate change. We therefore use a corrected value $\bar{r}_{\text {corr }}$, defined as the value of $\bar{r}$ that has the same significance with respect to a uniform Poisson process that the measured $\bar{r}$ has with respect to the empirical distribution.

$$
F_{\text {Poiss }}\left(\bar{r}_{\text {corr }}\right)=F_{\text {sim }}(\bar{r}),
$$


where $F_{\text {Poiss }}$ is the cumulative distribution function of $\bar{r}$ for a uniform Poisson process, and $F_{\text {sim }}$ is the model cumulative distribution function from the simulations (Fig. 7). Average rate change $\delta \lambda_{50}$ and confidence bounds $\delta \lambda_{05}$ and $\delta \lambda_{95}$ can be calculated from $\bar{r}_{\text {corr }}$ using the simple Poissonian stepchange model (Appendix A). Note that the model distribution in Figure 7 is only applicable for this particular subgroup of the data. The model distribution of $\bar{r}_{\text {sim }}$ must be recalculated for each particular subgroup.

\section{Results: Nonstationary Omori Reference}

\section{Global ISC Catalog}

We again apply the interevent time statistic to the global ISC catalog, but this time compute significance with respect to the nonstationary Omori reference model that accounts for aftershocks of major events. The post-seismic triggering signal reflected by the statistic $\left(p_{r}=0.37\right.$, Table 4$)$ is reduced using the more accurate reference model $\left(p_{r}=0.79\right.$, Table 5), as expected. Conversely, the precursory signal associated with the most recent trigger event prior to each great earthquake is enhanced ( $p_{r}=0.02$, Table 5). This result is suggestive, but not definitive, of a triggered rate increase at the site of at least some impending great earthquakes, as discussed in more detail below.

We now discuss the information presented by the ISC triggering matrix in more detail (Fig. 8). The sensitivity of the triggering tests at individual sites is very low, due to the small target regions and poor catalog completeness at most sites. Using equation 2 to estimate the expected rate change for each trigger-target pair beyond a $10^{\circ}$ distance (mean $\delta \lambda_{\exp }=9 \%$ ), we determine that in only a single case do we have sufficient data to exclude the small rate changes anticipated by equation 2 in the approximately one-degree radius around the regional epicenter. Even a rate increase of $67 \%$ (chosen as a representative "large" rate increase) cannot be excluded in any individual case. The sensitivity of the statistic is improved however, by looking at the combined statistics for all triggers at a particular region, or the combined statistics of a particular trigger at all regions. Trigger-target pairs separated by less than $10^{\circ}$ are excluded from this combined analysis. We also exclude the 2004 Sumatra-Andaman earthquake when calculating joint triggering significance, because trig-

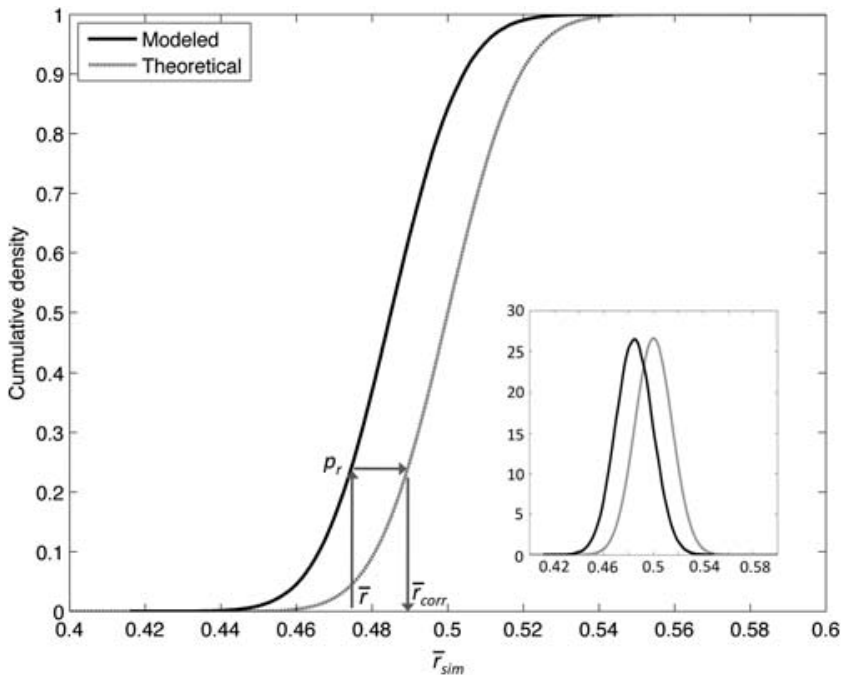

Figure 7. Modeled cumulative density function of $\bar{r}_{\text {sim }}$ based on a nonstationary Omori model rate (equation 4) that includes aftershocks of major regional and local events (solid black line). The dashed line shows the theoretical distribution for a stationary Poisson model (Appendix A). Inset: probability density functions. This particular example is for all trigger-target pairs greater than $10^{\circ}$ in distance, from the International Seismic Center (ISC) catalog. The arrows show the transformation from the observed statistic $\bar{r}$ to the corrected statistic $\bar{r}_{\text {corr }}$ (equation 5).

gering by the Sumatra earthquake is already reflected in the data for the 2004 Macquarie earthquake, due to the overlapping target time windows (see Triggers and Target Regions and Time Windows).

The joint triggering confidence for each trigger is depicted in the first column of the triggering matrix (Fig. 8). We should be able to resolve joint rate changes of $67 \%$ for nine of the triggers included in the ISC catalog (yellow boxes).

The joint triggering confidence for each region as a whole is depicted in the bottom row (Fig. 8). We do not detect significant triggering for any region and can rule out a $67 \%$ rate increase for 11 of the regions.

We now break the triggering matrix down into precursory and post-regional earthquake subgroups. The bottom right triangle of the triggering matrix is the place to look for precursory triggering. Triggering is detected above $90 \%$

Table 5

Statistical Significance: ISC Catalog-Omori Reference

\begin{tabular}{lccccccc}
\hline & $\bar{r}$ & $\bar{r}_{\text {corr }}$ & $m^{*}$ & $p_{r}$ & $p_{50}$ & $p_{90}$ & $\delta \lambda_{50}\left(\delta \lambda_{05,95}\right)[\%]$ \\
\hline All & 0.390 & 0.429 & 774 & $<\mathbf{1 0}^{-\mathbf{1 6}}$ & 0.47 & $\mathbf{0 . 0 2}$ & $\mathbf{5 3}(38,70)$ \\
All Distant & 0.496 & 0.510 & 468 & 0.76 & 0.78 & 0.79 & $-6(-17,8)$ \\
All Precursory & 0.498 & 0.504 & 218 & 0.58 & 0.88 & 0.73 & $-2(-19,19)$ \\
All Subsequent & 0.494 & 0.515 & 250 & 0.79 & 0.50 & 0.77 & $-9(-24,9)$ \\
1st Preceding & 0.422 & 0.386 & 25 & $\mathbf{0 . 0 2}$ & 0.75 & 0.23 & $\mathbf{1 0 1}(12,254)$ \\
1st Subsequent & 0.482 & 0.518 & 41 & 0.66 & 0.50 & 0.61 & $-10(-42,40)$ \\
\hline
\end{tabular}

*Sample size $m$ is the combined total number of local earthquake pairs $\left(t_{1}, t_{2}\right)$ in each subset, which can be greater than the number of great earthquake trigger-target pairs in the subset. 


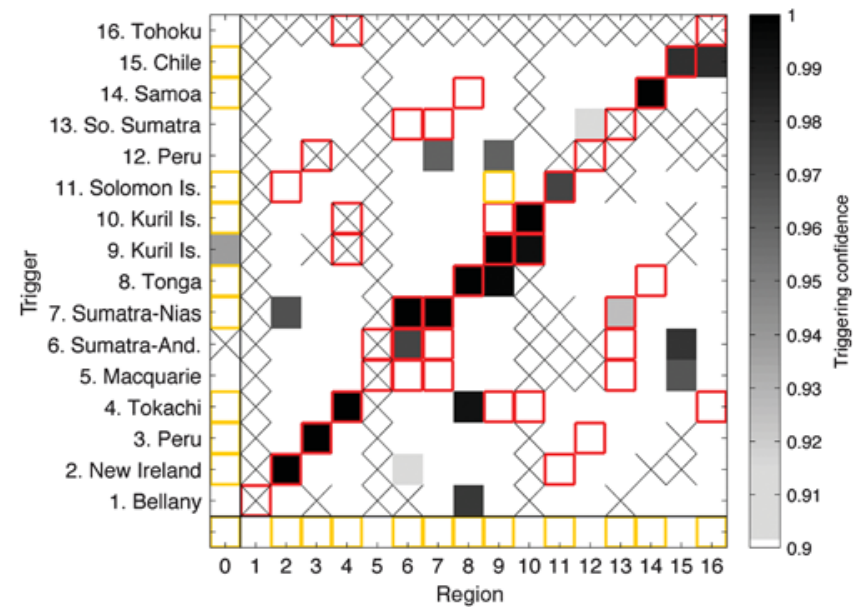

Figure 8. Triggering matrix for the International Seismic Center (ISC) catalog, using the nonstationary Omori reference model (compare with Fig. 4, using the uniform Poisson reference model). The triggering matrix shows regions with rate increases above $90 \%$ confidence (grayscale). Triggers are sorted by date along the $y$-axis and sorted as target regions on the $x$-axis. Yellow squares show where a rate increase of $67 \%$ could theoretically be detected or excluded. Red squares mark events within $10^{\circ}$ of each other. The far left column gives the combined triggering confidence for each trigger over all target regions (excluding those in red squares), and the bottom row shows the combined confidence for each region over all triggers (excluding red squares). The bottom left square gives the triggering confidence for the entire catalog. Crosses indicate no data for that trigger-target pair.

confidence at seven sites beyond a $10^{\circ}$ distance (Fig. 8). (Recall from the Methods Revisited section that the Macquarie 2004 and Sumatra 2004 detection successes at the site of the 2010 Chile quake count as a single case.) These seven cases of distant triggering cannot be considered significant in the context of multiple detection attempts, however, with $p_{90}=$ 0.73 (Table 5).

In the post-seismic, upper left triangle of the triggering matrix, we identify five instances of remote triggering. These five cases are not significant given the number of detection attempts $\left(p_{90}=0.77\right)$.

The estimated rate change (and 95\% confidence bounds) for the greater than $10^{\circ}$ distant group as a whole (pre- and post-seismic) is $\delta \lambda=-6 \%(-17,8)$. In the precursory group, the best estimate for triggered rate change is $\delta \lambda=-2 \%$ $(-19,19)$. In the post-regional earthquake group, the best estimate for triggered rate change is $\delta \lambda=-9 \%(-24,9)$.

In the First Preceding great earthquake subset, there does appear to be significant triggering, as mentioned above. The triggering matrix (Fig. 8) shows probable triggering by the 2006 Tonga earthquake at the site of the 2006 Kuril earthquake, with a confidence level of $99.8 \%$. There is also probably triggering by the 2010 Chile earthquake at the site of the impending 2011 Tohoku-Oki earthquake, with a confidence level of $98.0 \%$, two of the highest confidence levels in the triggering matrix. The joint significance for this subgroup is $p_{r}=0.02$. However, the other two statistics do not show

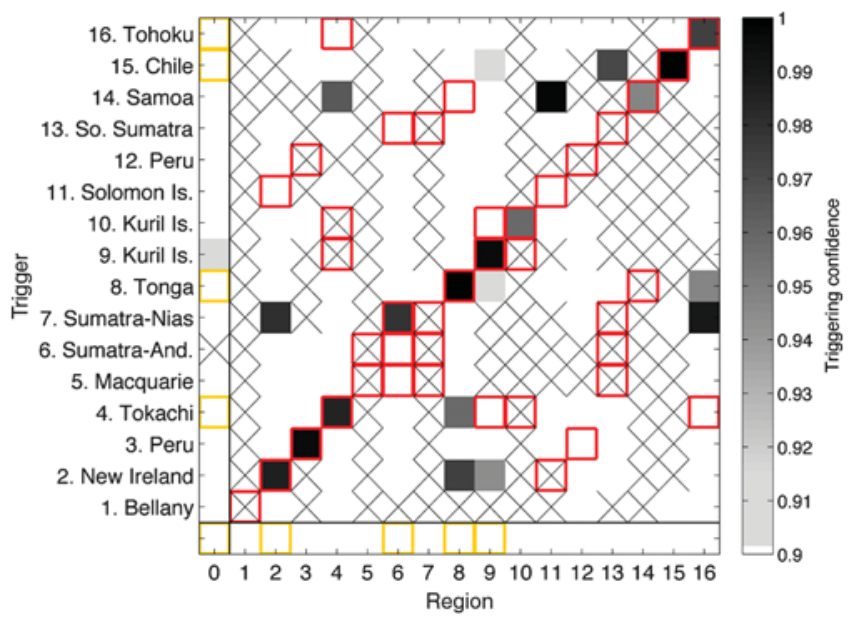

Figure 9. Triggering matrix for the Preliminary Determination of Epicenters (PDE) catalog, using a nonstationary Omori reference model. See caption of Figure 8 for details.

a significant signal, with no more detection success than expected by chance $\left(p_{90}=0.23\right)$ and no more apparent increases than decreases in the calculated rate changes $\left(p_{50}=0.75\right)$.

\section{Global PDE Catalog}

The triggering calculations are again conducted for the global PDE catalog (Fig. 9). This catalog is less complete than the ISC, with less robust statistics, but it does capture the two most recent triggers. We consider the results of the ISC catalog to be definitive where they replace PDE data. The PDE catalog reveals a pattern similar to the ISC catalog. The statistics are summarized in Figure 9 and Table 6.

The PDE data also do not show significant triggering for quakes $>10^{\circ}$ as a whole, with $p_{r}=0.89$, nor for any of the precursory or subsequent earthquake subgroups (Table 6). In contrast to the ISC catalog, triggering is not identified for the sub-population containing only the First Preceding earthquake in each region $\left(p_{r}=0.42\right)$.

Computing the joint significance region-by-region, we detect apparent triggering in none of the epicentral regions. We can rule out a $67 \%$ rate increase for one-third of the target regions.

The estimated rate change (and 95\% confidence bounds) for the greater than $10^{\circ}$ distance group as a whole is $\delta \lambda=$ $-13 \%(-28,5)$. In the precursory group, the best estimate for triggered rate change is $\delta \lambda=-30 \%(-49,-2)$. The statistics therefore indicate systematic triggered rate decreases in this subgroup. This is probably a catalog artifact, as this observation is not reproduced in the ISC or JMA catalogs. In the post-regional earthquake group, the best estimate for triggered rate change is $\delta \lambda=-4 \%(-23,21)$. We therefore conclude that the PDE catalog also contains no evidence for systematic earthquake triggering by distant great earthquakes at the site of other impending great earthquakes. 


\section{JMA Catalog}

Finally, we examine the JMA catalog, which is the most complete in its region of coverage. The catalog covers only the regions of the 2004 Tokachi-Oki and 2011 Tohoku-Oki epicenters, where it is complete to magnitude $M_{C}=1.3$.

Triggering at sites greater than a $10^{\circ}$ distance is only observed above $90 \%$ confidence for a single trigger: 2001 Peru, but at both target sites in Japan (Fig. 10). However, this detection success is not significant given the number of detection attempts $\left(p_{90}=0.79\right)$. Neither the precursory nor post-regional earthquake subgroups show significant triggering, with an estimated rate change very near zero for each (Table 7). For the precursory group, we get an estimated rate change (with $95 \%$ confidence bounds) $\delta \lambda=0 \%(-6,7)$.

It should be noted that, in particular, the 2010 Chile earthquake is not identified as a strong trigger at the site of the impending 2011 Tohoku-Oki earthquake (Fig. 10), in contrast to the ISC catalog (Fig. 8). The triggering signal reflected in the ISC catalog is therefore not corroborated by the JMA cata$\log$, despite its superior completeness and sample size.

Thanks to the higher completeness of the JMA catalog, we can say with $95 \%$ confidence that the triggered rate change in both target regions in Japan is smaller than that expected from equation (2), based on triggering in California. This confirms previous studies that have found Japan to be less triggerable than California (Harrington and Brodsky, 2006; van der Elst and Brodsky, 2010).

\section{Discussion}

\section{Triggering at Sites of Impending Large Earthquakes}

The rate of recent great $M_{\mathrm{w}} \geq 8$ earthquakes has increased with respect to the preceding century by $50 \%-160 \%$, depending on the time window and the method used to remove conventional aftershocks. Using the ISC catalog, the best estimate for the systematic rate increase at the site of impending great earthquakes due to all preceding great earthquakes is $\delta \lambda=-2 \%$ with lower and upper $95 \%$ confidence bounds of $-19 \%$ and $19 \%$. There is a suggestive signal in the ISC catalog for the subset of events that fall just prior to each regional great earthquake in the sequence (Table 5). However, the signal is dominated by a single instance, the 2010 Chile earthquake at the site of the 2011 Tohoku-Oki earthquake (Fig. 8), and this signal is not corroborated by

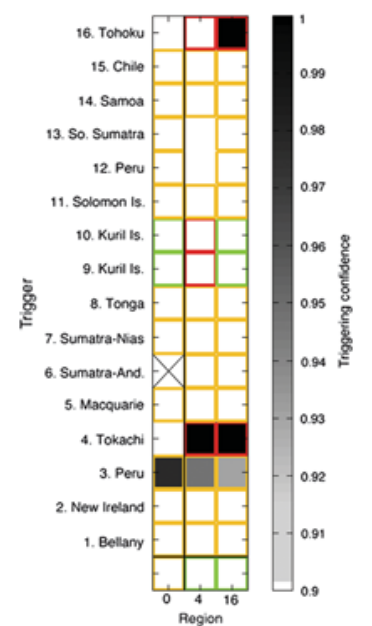

Figure 10. Triggering matrix for the Japan Meteorological Agency (JMA) catalog, using a nonstationary Omori reference model, showing the Tokachi-Oki and Tohoku-Oki regions only. Green squares show where the expected rate change (equation 2) should be resolvable, and yellow squares show where a $67 \%$ rate change should be resolvable. See caption of Figure 9 for other features.

the higher-quality JMA dataset (Fig. 10), making the observation inconclusive.

We therefore conclude that there is no evidence for large or systematic triggered rate increases at the sites of impending great $M_{\mathrm{w}} \geq 8$ earthquakes. If the occurrence of some great earthquakes is to be explained by a long-range triggering process, it must do so only rarely, or without systematically affecting the rates of earlier, smaller earthquakes at the time of the trigger.

It is more difficult to rule out triggering on a less systematic, case-by-case basis, as even doublings of rates near the epicenters of most impending earthquakes cannot be excluded with high confidence due to the scarcity of data in most places. Only the JMA catalog has adequate coverage to detect systematic triggering at the level expected from the California productivity scaling for a few cases (Fig. 10), and where this coverage exists, triggering is not detected.

\section{Magnitude of Completeness Required to Detect Small Rate Changes}

We are reasonably confident that the earthquake catalogs do not show systematic large triggered rate changes

Table 6

Statistical Significance: PDE Catalog-Omori Reference

\begin{tabular}{lccrcccc}
\hline & $\bar{r}$ & $\bar{r}_{\text {corr }}$ & \multicolumn{1}{c}{$m$} & $p_{r}$ & $p_{50}$ & $p_{90}$ & $\delta \lambda_{50}\left(\delta \lambda_{05,95}\right)[\%]$ \\
\hline All & 0.417 & 0.440 & 368 & $\mathbf{1 0}^{-4}$ & 0.61 & $\mathbf{1 0}^{-\mathbf{3}}$ & $\mathbf{4 3}(22,67)$ \\
All Distant & 0.513 & 0.523 & 231 & 0.89 & 0.96 & 0.25 & $-13(-28,5)$ \\
All Precursory & 0.545 & 0.558 & 77 & 0.96 & 0.99 & 0.24 & $-30(-49,-2)$ \\
All Subsequent & 0.497 & 0.506 & 154 & 0.61 & 0.66 & 0.46 & $-4(-23,21)$ \\
1st Preceding & 0.512 & 0.481 & 10 & 0.42 & 0.66 & 0.47 & $12(-54,169)$ \\
1st Subsequent & 0.496 & 0.519 & 18 & 0.61 & 0.50 & 1.00 & $-11(-54,74)$
\end{tabular}


capable of explaining recent large earthquake rates. What might smaller sequences, such as those expected from other continental triggering studies, tell us about earthquake processes? We now use the framework developed in this paper to estimate the magnitude of completeness needed to routinely identify small triggered sequences in subduction zones, where most great earthquakes occur. In most cases, the current network coverage is insufficient to detect the small rate changes expected from established scaling laws (equation 2). Figure 11 shows the corrected interevent time statistic $\bar{r}_{\text {corr }}$ (equation 5), along with the expected value of $\bar{r}$ (equation A8) corresponding to the rate change $\delta \lambda$ expected from equation (2), as well as the value of $\bar{r}$ required to exceed the $90 \%$ confidence threshold given the available number of observations in the ISC catalog. In no case is the number of observations (catalog completeness level) adequate to establish the expected rate change at the $90 \%$ confidence level.

We now estimate the number of observations that would be required to consistently detect rate changes expected from the California scaling (equation 2). If we assume that earthquakes follow the Gutenberg-Richter magnitude frequency scaling with $b=1$, this required sample size can be expressed in terms of the required completeness magnitude. We estimate the required catalog completeness level for (1) the catalog as a whole, (2) each target region as a whole, and (3) for each individual trigger-target pair. This calculation is slightly dependent on the method used to calculate the current magnitude of completeness. The required magnitudes reported below are calculated using a completeness threshold of $90 \%$ (Wiemer and Wyss, 2000), consistent with the magnitude cutoff used in the rest of this study. To show that the projections are robust within $\sim 0.1$ magnitude units, we also report required completeness magnitudes calculated with a $95 \%$ threshold in Table 8.

The mean expected rate change, averaged over all distant trigger-target pairs, is $\delta \lambda_{\exp }=9 \%$. To have $95 \%$ confidence that this rate change would produce a positive statistical signal in the ISC catalog (i.e., an interevent time statistic $\bar{r}<0.5$ ), we would need only a relatively marginal improvement in the magnitude of $90 \%$ completeness from 3.6 to 3.5 (Table 8). For the $95 \%$ completeness threshold, the magnitude of completeness would require improvement from 4.0 to 3.6.

On a regional basis, the required magnitude of completeness is dependent on the average seismicity rate, with less active regions requiring a proportionally lower magnitude of completeness to produce the same sample size (Table 8). The

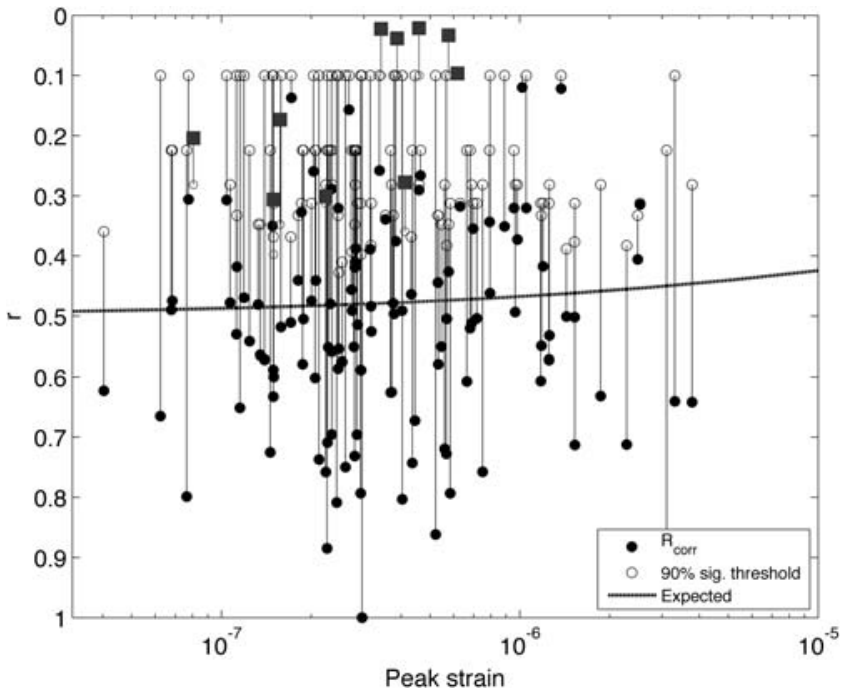

Figure 11. Observed $\bar{r}_{\text {corr }}$ (filled circles) compared with the $\bar{r}$ needed to exceed the $90 \%$ confidence threshold given the number of samples (open circles), for trigger-target pairs greater than $10^{\circ}$ in distance, as a function of trigger strain. Filled squares are cases where the observed rate change exceeds $90 \%$ confidence. The dashed line shows the expected $\bar{r}$ and associated rate change from equation 2. In no case is the expected rate change (dashed line) above the threshold for significance (open circles) for isolated trigger-target pairs.

required magnitude of $90 \%$ completeness for detecting the median expected rate change on a joint regional basis is 2.1 . This completeness level is obtained only by the JMA catalog.

On a case-by-case basis (Fig. 11), the magnitude of completeness would have to be considerably lower. The median required magnitude of completeness, that is, the level required to detect distant triggering at half of all sites (median rate change $\delta \lambda_{\exp }=7 \%$ ) is $M_{C}=0.6$. This level of completeness is likely impossible without dense offshore networks of seismic stations.

\section{Limitations}

Great earthquake interactions are only considered here in terms of local cascade dynamics. Other possibilities for linking great earthquakes might be: (1) triggering a deep slow slip event (Shelly et al., 2011) that then initiates a great earthquake at the down-dip extent of locking; or (2) viscous stress transfer in the lower crust and upper mantle, operating on a much longer timescale than the passage of seismic waves (Freed and Lin, 1998; Pollitz et al., 1998). A careful search

Table 7

Statistical Significance: JMA Catalog-Omori Reference

\begin{tabular}{lccccccc}
\hline & $\bar{r}$ & $\bar{r}_{\text {corr }}$ & $m$ & $p_{r}$ & $p_{50}$ & $p_{90}$ & $\delta \lambda_{50}\left(\delta \lambda_{05,95}\right)[\%]$ \\
\hline All & 0.473 & 0.466 & 3958 & $<\mathbf{1 0}^{-\mathbf{1 6}}$ & 0.70 & 0.21 & $\mathbf{2 3}(18,29)$ \\
All Distant & 0.496 & 0.499 & 2534 & 0.42 & 0.80 & 0.41 & $1(-5,7)$ \\
All Precursory & 0.497 & 0.499 & 2090 & 0.46 & 0.25 & 0.23 & $0(-6,7)$ \\
All Subsequent & 0.494 & 0.494 & 444 & 0.33 & 0.97 & 0.77 & $4(-9,19)$ \\
& & & & & & & \\
\hline
\end{tabular}


Table 8

Required ISC Magnitude of Completeness to Detect Expected Triggering

\begin{tabular}{lccccc}
\hline \multicolumn{1}{c}{ Region } & $\begin{array}{c}\text { Median Expected } \\
\text { Rate Change } \\
\delta \lambda_{\exp }\end{array}$ & $\begin{array}{c}\text { Number of Earthquake } \\
\text { Pairs in Catalog } \\
(90 \% / 95 \%)^{*}\end{array}$ & $\begin{array}{c}\text { Number of Pairs Required } \\
\text { to Resolve Rate } \\
\text { Change } \delta \lambda_{\text {exp }}\end{array}$ & $\begin{array}{c}\text { Completeness } \\
\text { Magnitude } M_{C} \\
(90 \% / 95 \%)^{*}\end{array}$ & $\begin{array}{c}M_{\mathrm{C}} \text { Required to Resolve } \\
\text { Rate Change } \delta \lambda_{\text {exp }} \\
(90 \% / 95 \%)^{a *}\end{array}$ \\
\hline Balleny Island & 0.06 & $0 / 0$ & 1596 & $4.1 / 5.5$ & $-1-$ \\
New Ireland & 0.07 & $41 / 10$ & 959 & $3.6 / 4.3$ & $2.2 / 2.3$ \\
Peru & 0.08 & $22 / 20$ & 842 & $3.6 / 3.8$ & $2.0 / 2.2$ \\
Tokachi-Oki & 0.07 & $26 / 26$ & 1134 & $3.5 / 3.5$ & $1.9 / 1.9$ \\
Macquarie & 0.08 & $0 / 0$ & 831 & $5.1 / 5.1$ & $-1-$ \\
Sumatra-Andaman & 0.11 & $60 / 48$ & 468 & $3.8 / 4.0$ & $2.9 / 3.0$ \\
Sumatra-Nias & 0.10 & $37 / 4$ & 519 & $3.7 / 4.6$ & $2.6 / 2.5$ \\
Tonga & 0.07 & $51 / 41$ & 1020 & $3.8 / 4.0$ & $2.5 / 2.6$ \\
Kuril & 0.06 & $67 / 21$ & 1689 & $3.5 / 4.2$ & $2.1 / 2.3$ \\
Kuril & 0.07 & $2 / 2$ & 1123 & $3.6 / 3.7$ & $0.9 / 1.0$ \\
Solomon Islands & 0.07 & $25 / 12$ & 995 & $3.6 / 4.0$ & $2.0 / 2.1$ \\
Peru & 0.07 & $19 / 0$ & 1204 & $3.5 / 6.1$ & $1.7 /-$ \\
South Sumatra & 0.08 & $29 / 0$ & 920 & $3.6 / 7.6$ & $2.1 /-$ \\
Samoa & 0.07 & $34 / 31$ & 1011 & $3.7 / 3.8$ & $2.2 / 2.3$ \\
Chile & 0.09 & $15 / 15$ & 663 & $3.7 / 3.7$ & $2.1 / 2.1$ \\
Tohoku-Oki & 0.14 & $55 / 55$ & 293 & $3.5 / 3.5$ & $2.8 / 2.8$ \\
Combined & $\mathbf{0 . 0 9}$ & $\mathbf{4 8 3 / 2 8 5}$ & $\mathbf{6 6 4}$ & $\mathbf{3 . 6 / 4 . 0}$ & $\mathbf{3 . 5 / 3 . 6}$ \\
\hline
\end{tabular}

*Goodness-of-fit test threshold used to determine completeness magnitude.

for remotely triggered tectonic tremor down-dip of impending earthquakes could evaluate whether great earthquakes are linked through triggered deep slip.

We have looked at the largest magnitude earthquakes with the greatest triggering potential. The largest triggers are the most likely places to look for a response, but smaller magnitude triggers may be cumulatively just as important in triggering distant rate changes, due to their proportionally greater number (Helmstetter et al., 2005). Triggering may also be influenced by precise stress orientations of dynamic waves, which vary with fault geometry and relative location of trigger events and target nucleation zones (Hill, 2008). In some cases, a smaller nearby event may be more efficient for triggering at a particular target.

\section{Conclusion}

We have systematically measured triggered rate changes at the sites of $16 M_{\mathrm{w}} \geq 8$ earthquakes, at the times of other $M_{\mathrm{w}} \geq 8$ earthquakes, during the high-great earthquake rate period between 1998 and 2011. We determine that there is no evidence that $M_{\mathrm{w}} \geq 8$ earthquakes trigger precursory activity at the site of other impending $M_{\mathrm{w}} \geq 8$ earthquakes on a systematic basis, and triggering on a scale capable of explaining a $50 \%-160 \%$ increase in the rate of $M_{\mathrm{w}} \geq 8$ earthquakes can be ruled out with $95 \%$ confidence. More limited triggering on a case-by-case basis cannot be ruled out with high confidence.

The data are, however, consistent with expected rate changes given the amplitude of seismic shaking at the target sites, although an improvement in completeness magnitude from 3.6 to 3.5 would be required to definitively establish small rate changes.
There is no apparent increase in regional triggering susceptibility that might serve as a precursory signal of impending earthquakes, at least using current catalogs. However, regional networks with a magnitude of completeness down to 2.0 would be required to adequately resolve expected triggered rate changes on a region-by-region basis.

\section{Data and Resources}

Several earthquake catalogs were used in this study. ISC data through 2007 were obtained on CDs from the ISC, and supplemented with data through November 2009 via ftp from http://www.isc.ac.uk (last accessed August 2012). The PDE catalog was downloaded via ftp from the U.S. Geological Survey website, http://www.earthquake.usgs.gov/ research/data/pde.php (last accessed August 2012). The JMA catalog was downloaded from the agency's website at http://www.hinet.bosai.go.jp (last accessed 22 November 2011, account required). The USGS PAGER catalog, used to define the list of $M_{\mathrm{w}} \geq 8$ earthquakes, is available from http:// www.earthquake.usgs.gov/research/data. Seismograms were obtained from the IRIS Data Management Center at http:// www.iris.edu and processed in SAC. Many seismographic networks contributed data through IRIS.

\section{Acknowledgments}

We are grateful to Andy Michael, an anonymous reviewer, and the Associate Editor Morgan Page for their constructive comments that helped to improve the paper. This paper benefited from exchanges of perspectives and preprints with several parties, including Peter Shearer, Andy Michael, Eric G. Daub, and Paul A. Johnson. This work was supported by NSF Grants EAR-0838598 (EEB, NJV) and EAR-0635570 (TL). 


\section{References}

Ammon, C. J., T. Lay, and D. W. Simpson (2010). Great earthquakes and global seismic networks, Seismol. Res. Lett. 81, no. 6, 965-971.

Anderson, J. G., J. N. Brune, J. N. Louie, Y. H. Zeng, M. Savage, G. Yu, Q. B. Chen, and D. Depolo (1994). Seismicity in the Western Great Basin apparently triggered by the Landers, California, Earthquake, 28 June 1992, Bull. Seismol. Soc. Am. 84, no. 3, 863-891.

Beeler, N. M., and D. A. Lockner (2003). Why earthquakes correlate weakly with the solid Earth tides: Effects of periodic stress on the rate and probability of earthquake occurrence, J. Geophys. Res. 108, no. B8, doi: $10.1029 / 2001 \mathrm{jb} 001518$.

Brodsky, E. E. (2009). The 2004-2008 worldwide superswarm (abstract S53B-06), Eos Trans. AGU 90, no. 52 (Fall Meet. Suppl.), S53B-06.

Casella, G., and R. L. Berger (2002). Statistical Inference, Second Ed., Vol. xxviii, Thomson Learning/Pacific Grove, Australia/California, $660 \mathrm{pp}$.

Felzer, K. R., and E. E. Brodsky (2005). Testing the stress shadow hypothesis, J. Geophys. Res. 110, no. B5, B05S09.

Felzer, K. R., R. E. Abercrombie, and G. Ekstrom (2004). A common origin for aftershocks, foreshocks, and multiplets, Bull. Seismol. Soc. Am. 94, no. $1,88-98$.

Felzer, K. R., T. W. Becker, R. E. Abercrombie, G. Ekstrom, and J. R. Rice (2002). Triggering of the $1999 M_{\mathrm{w}} 7.1$ Hector Mine earthquake by aftershocks of the $1992 M_{\mathrm{w}} 7.3$ Landers earthquake, J. Geophys. Res. 107, no. B9, doi: 10.1029/2001jb000911.

Freed, A. M., and J. Lin (1998). Time-dependent changes in failure stress following thrust earthquakes, J. Geophys. Res. 103, no. B10, 24,39324,409 .

Gomberg, J. (2001). The failure of earthquake failure models, J. Geophys. Res. 106, no. B8, 16,253-16,263.

Gomberg, J., and P. Johnson (2005). Seismology-Dynamic triggering of earthquakes, Nature 437, no. 7060, 830.

Gomberg, J., P. Bodin, K. Larson, and H. Dragert (2004). Earthquake nucleation by transient deformations caused by the $M 7.9$ Denali, Alaska, earthquake, Nature 427, no. 6975, 621-624.

Grinstead, C. M., and J. L. Snell (1997). Introduction to Probability, American Mathematical Society available at http://www.ams.org/ bookstore-getitem/item=IPROB-S, 291-304.

Harrington, R. M., and E. E. Brodsky (2006). The absence of remotely triggered seismicity in Japan, Bull. Seismol. Soc. Am. 96, no. 3, 871-878.

Helmstetter, A., Y. Y. Kagan, and D. D. Jackson (2005). Importance of small earthquakes for stress transfers and earthquake triggering, J. Geophys. Res. 110, no. B5, B05S08.

Hill, D. P. (2008). Dynamic stresses, Coulomb failure, and remote triggering, Bull. Seismol. Soc. Am. 98, no. 1, 66-92.

Hill, D. P., and S. G. Prejean (2007). Dynamic triggering, in Earthquake Seismology, H. Kanamori (Editor), Treatise on Geophysics, Schubert, G. (Editor in Chief), Vol. 4, Elsevier, Amsterdam, 258-288.

Lay, T., and T. C. Wallace (1995). Modern Global Seismology, Academic Press, San Diego, 382 pp.

Lei, X. L., C. D. Xie, and B. H. Fu (2011). Remotely triggered seismicity in Yunnan, southwestern China, following the $2004 M_{\mathrm{w}} 9.3$ Sumatra earthquake, J. Geophys. Res. 116, B08303, doi: 10.1029/2011JB008245.

Meng, L., J.-P. Ampuero, J. Stock, Z. Duputel, Y. Luo, and V. C. Tsai (2012). Earthquake in a maze: Compressional rupture branching during the $2012 M_{\mathrm{w}}$ 8.6 Sumatra earthquake, Science 337, no. 6095, 724-726.

Michael, A. J. (2011). Random variability explains apparent global clustering of large earthquakes, Geophys. Res. Lett. 38, L21301, doi: 10.1029/2011GL049443.

Mignan, A., and J. Woessner (2012). Estimating the magnitude of completeness for earthquake catalogs, Community Online Resource for Statistical Seismicity Analysis, doi: 10.5078/corssa-00180805.

Nalbant, S. S., S. Steacy, K. Sieh, D. Natawidjaja, and J. McCloskey (2005). Earthquake risk on the Sunda trench, Nature 435, no. 7043, $756-757$.
Ogata, Y. (1992). Detection of precursory relative quiescence before great earthquakes through a statistical model, J. Geophys. Res. 97, no. B13, $19,845-19,871$.

Parsons, T., and A. A. Velasco (2011). Absence of remotely triggered large earthquakes beyond the mainshock region, Nature Geosci. 4, no. 5, 312-316.

Peng, Z. G., D. P. Hill, D. R. Shelly, and C. Aiken (2010). Remotely triggered microearthquakes and tremor in central California following the $2010 M_{\mathrm{w}} 8.8$ Chile earthquake, Geophys. Res. Lett. 37, doi: 10.1029/ 2010GL045462.

Pollitz, F. F., R. Burgmann, and B. Romanowicz (1998). Viscosity of oceanic asthenosphere inferred from remote triggering of earthquakes, Science 280, no. 5367, 1245-1249.

Rubinstein, J. L., G. C. Beroza, J. Brown, M. Brudzinski, J. Gomberg, S. Malone, D. H. Oppenheimer, Z. G. Peng, S. G. Prejean, H. M. Savage, D. R. Shelly, A. Wech, and M. West (2011). Widespread triggering of earthquakes and tremor by the 2011 M 9.0 off-Tohoku earthquake, Seismol. Res. Lett. 82, no. 2, 273-361.

Savage, H. M., and C. Marone (2008). Potential for earthquake triggering from transient deformations, J. Geophys. Res. 113, no. B5, B05302, doi: $10.1029 / 2007 j b 005277$.

Shearer, P. M., and P. B. Stark (2011). Global risk of big earthquakes has not recently increased, Proc. Natl. Acad. Sci. 109, no. 3, 717-721.

Shelly, D. R., Z. G. Peng, D. P. Hill, and C. Aiken (2011). Triggered creep as a possible mechanism for delayed dynamic triggering of tremor and earthquakes, Nature Geosci. 4, no. 6, 384-388.

Uspensky, J. B. (1937). Introduction to Mathematical Probability, McGraw-Hill, New York, 277 pp.

Van der Elst, N. J., and E. E. Brodsky (2010). Connecting near-field and far-field earthquake triggering to dynamic strain, J. Geophys. Res. 115, B07311, doi: 10.1029/2009JB006681.

Velasco, A. A., S. Hernandez, T. Parsons, and K. Pankow (2008). Global ubiquity of dynamic earthquake triggering, Nature Geosci. 1, no. 6, 375-379.

West, M., J. J. Sanchez, and S. R. McNutt (2005). Periodically triggered seismicity at Mount Wrangell, Alaska, after the Sumatra earthquake, Science 308, no. 5725, 1144-1146.

Wiemer, S., and M. Wyss (2000). Minimum magnitude of completeness in earthquake catalogs: Examples from Alaska, the western United States, and Japan, Bull. Seismol. Soc. Am. 90, no. 4, 859-869.

Wiseman, K., and R. Burgmann (2011). Stress and seismicity changes on the Sunda megathrust preceding the $2007 M_{\mathrm{w}} 8.4$ Earthquake, Bull. Seismol. Soc. Am. 101, no. 1, 313-326.

Wu, C. Q., Z. G. Peng, W. J. Wang, and Q. F. Chen (2011). Dynamic triggering of shallow earthquakes near Beijing, China, Geophys. J. Int. 185, no. $3,1321-1334$.

\section{Appendix A}

\section{Distribution of the Sample Mean of $r$}

We use the sampling distribution of the mean interevent time ratio $\bar{r}$ to calculate the significance of $\bar{r}$ with respect to a Poisson process, as well as to determine the minimum rate increase at which the Poisson process could be rejected. The distribution of $\bar{r}$ in the case of zero rate change is known, as $r$ is uniform on $[0,1]$. The distribution of the sum $s=m \bar{r}$ of a sample of uniform distributed random variables is (Uspensky, 1937)

$$
f_{S}(s \mid m)=\sum_{k=0}^{m}(-1)^{k} \frac{m}{k !(m-k) !}(s-k)^{m-1} u(s-k) .
$$

Here, $u$ is the unit step function. In this appendix, we use the convention that distributions are subscripted by their 
applicable variable in uppercase. The distribution of the mean is the distribution (A1) rescaled over the range $[0,1]$, that is,

$$
\begin{gathered}
f_{\bar{R}}(\bar{r})=m f_{S}(m \bar{r}) . \\
f_{\bar{R}}(\bar{r} \mid m)=\sum_{k=0}^{m}(-1)^{k} \frac{m^{2}}{k !(m-k) !}(m \bar{r}-k)^{m-1} u(m \bar{r}-k) .
\end{gathered}
$$

This distribution very rapidly approaches the normal distribution as $m$ increases (Fig. A1).

The distribution of $\bar{r}$ for an arbitrary rate change $\delta \lambda$ is required to compute confidence bounds, that is, the minimum and maximum rate changes that could pass the Poisson hypothesis test. Van der Elst and Brodsky (2010) derived the expectation of $r$ for a step change in a Poisson process. Here, we give the full distribution function.

The distribution of $r$ (defined in equation 1) with $t_{1}$ and $t_{2}$ drawn from distinct Poisson processes with intensities $\lambda_{1}$ and $\lambda_{2}$, respectively, is

$$
f_{R}\left(r \mid \lambda_{1}, \lambda_{2}\right)=\frac{\lambda_{1} \lambda_{2}}{\left[(1-r) \lambda_{1}+r \lambda_{2}\right]^{2}} .
$$

This equation follows from transforming the joint probability distribution of two independent, exponentially distributed times $t_{1}$ and $t_{2}$, with means $\lambda_{1}^{-1}$ and $\lambda_{2}^{-1}$, into a function of the random variable $r$ (van der Elst and Brodsky, 2010).

Defining fractional rate change as

$$
\delta \lambda \equiv \frac{\lambda_{2}-\lambda_{1}}{\lambda_{1}},
$$

and substituting (A5) into (A4) gives

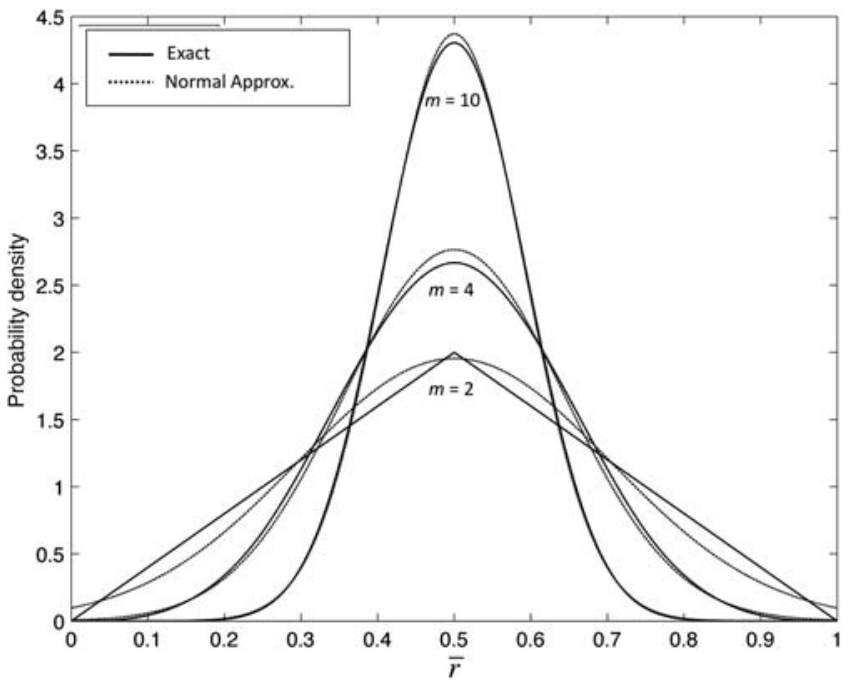

Figure A1. Distribution of the sample mean $\bar{r}$ for a stationary Poisson process (equation A3), for various sample sizes $m$. Solid lines are the exact solution given by (equation A3), and the dashed lines show the approximation using a normal distribution.

$$
f_{R}(r \mid \delta \lambda)=\frac{\delta \lambda+1}{(1+\delta \lambda \cdot r)^{2}}
$$

The distribution of the sample mean $\bar{r}$ (i.e., the first moment) as a function of fractional rate change $\delta \lambda$ and sample size $m$ cannot be obtained analytically, as the moment generating function for (A6) is expressible only with special functions. For small samples $(m<10)$, we use numerical convolution to obtain the distribution and cumulative distribution of the sum $s$ (Grinstead and Snell, 1997) and then rescale this distribution (equation A2) to obtain the distribution of the mean $\bar{r}$.

For larger samples $(m>10)$, we take advantage of the central limit theorem to obtain an analytical approximation of the distribution of the mean. The central limit theorem guarantees that the distribution of the mean of any random variable with mean $\mu$ and finite variance $\sigma^{2}$ approaches a normal distribution with mean $\mu$ and variance $\sigma^{2} / m$ as sample size $m$ increases (Casella and Berger, 2002). The mean of $r$ as a function of $\delta \lambda$ can be found by computing the expectation from (A6):

$$
\mu_{r}(\delta \lambda)=\int_{0}^{1} r f_{R}(r \mid \delta \lambda) d r
$$

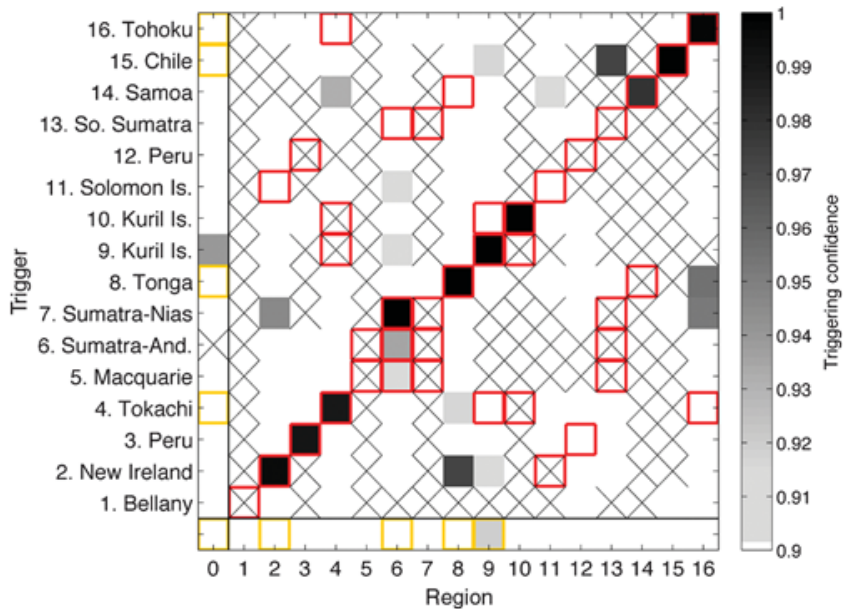

Figure B1. Triggering matrix for the Preliminary Determination of Epicenters (PDE) catalog, with significance measured with respect to a stationary, uniform Poisson process. The triggering matrix shows regions with rate increases above a $90 \%$ confidence level (grayscale). Triggers are sorted by date along the $y$-axis and sorted as target regions on the $x$-axis. Yellow squares show where a rate increase of $67 \%$ could theoretically be detected or excluded. Red squares mark events within $10^{\circ}$ of each other. The far left column gives the combined triggering confidence for each trigger over all target regions (excluding those in red squares), and the bottom row shows the combined confidence for each region over all triggers (excluding red squares). The bottom left square gives the triggering confidence for the entire catalog. Crosses indicate no data for that trigger-target pair. 


$$
\mu_{r}(\delta \lambda)=\frac{1}{\delta \lambda^{2}}[(\delta \lambda+1) \ln (\delta \lambda+1)-\delta \lambda]
$$

and the variance (expressed as a function of the mean for simplicity) is

$$
\sigma_{r}^{2}(\delta \lambda)=\frac{1}{\delta \lambda}\left[1-2 \mu_{r}\right]-\mu_{r}^{2}
$$

In the limit of $\delta \lambda=0$, the mean $\mu_{r}$ and variance $\sigma_{r}^{2}$ can be shown to be $1 / 2$ and $1 / 12$, respectively, consistent with a uniform distribution on $[0,1]$. We determine that the normal approximation using (A8) and (A9) is very good for $m>10$ and $\delta \lambda<1$.

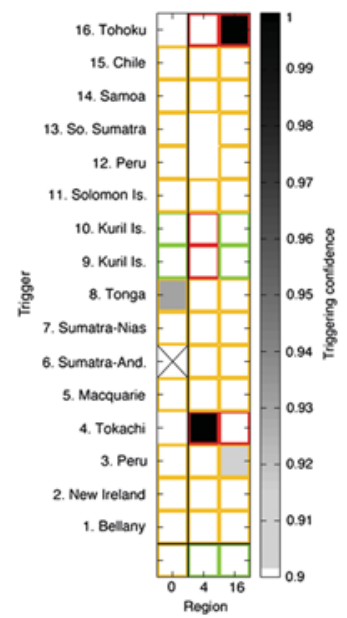

Figure B2. Triggering matrix for the Japan Meteorological Agency (JMA) catalog using a stationary, uniform Poisson reference model showing the Tokachi-Oki and Tohoku-Oki regions only. Green squares show where the expected rate change (equation 2) should be resolvable, and yellow squares show where a $67 \%$ rate change should be resolvable. See caption of Figure B1 for other features.
The significance of $\bar{r}$ is defined as the probability $p_{r}$ that a uniform random process would produce a smaller mean than that observed, by chance:

$$
p_{r} \equiv F_{\bar{R}}(\bar{r})
$$

where $F_{\bar{R}}$ is the cumulative distribution function. Using the normal approximation with (A8) and (A9),

$$
F_{\bar{R}}(\bar{r}) \cong \Phi\left[\bar{r} \mid \mu_{r}(0), \sigma_{r}^{2}(0) / m\right],
$$

where $\Phi$ is the cumulative normal distribution.

Inverting the statistic (A10) gives the confidence bounds on the rate change parameter $\delta \lambda$ (Casella and Berger, 2002). The maximum bound on the rate change $\delta \lambda$, at significance level $\alpha$ (e.g., 0.05), is the value of $\delta \lambda$ for which

$$
\alpha=F_{\bar{R}}(\delta \lambda \mid \bar{r}) \cong \Phi\left[\bar{r} \mid \mu_{r}(\delta \lambda), \sigma_{r}^{2}(\delta \lambda) / m\right] .
$$

Given $\bar{r}, m$, and $\alpha$, we solve iteratively for $\delta \lambda$. To obtain the minimum bound on $\delta \lambda$, we solve (A12) for $\alpha^{\prime}=1-\alpha$.

\section{Appendix B}

\section{Analysis of PDE and JMA Catalogs Using Uniform Poisson Reference}

See Figures B1 and B2, and Tables B1 and B2.

\section{Appendix C}

\section{Omori Model Parameters}

This appendix contains the parameters used in the nonstationary Omori reference models (equation 4). It also lists the

Table B1

Statistical Significance: PDE Catalog—Poisson Reference

\begin{tabular}{lcrcccc}
\hline & $r$ & \multicolumn{1}{c}{$m$} & $p_{r}$ & $p_{50}$ & $p_{90}$ & \multicolumn{1}{c}{$\delta \lambda_{50}\left(\delta \lambda_{05,95}\right)[\%]$} \\
\hline All & 0.417 & 368 & $\mathbf{1 0}^{-8}$ & 0.46 & $\mathbf{1 0}^{-4}$ & $\mathbf{6 5}(42,92)$ \\
All Distant & 0.513 & 231 & 0.75 & 0.94 & 0.25 & $-8(-123,12)$ \\
All Precursory & 0.545 & 77 & 0.92 & 0.99 & 0.41 & $-24(-45,5)$ \\
All Subsequent & 0.497 & 154 & 0.45 & 0.55 & 0.29 & $2(-19,28)$ \\
1st Preceding & 0.512 & 10 & 0.55 & 0.66 & 1.00 & $-7(-61,125)$ \\
1st Subsequent & 0.496 & 18 & 0.48 & 0.23 & 1.00 & $2(-47,98)$ \\
\hline
\end{tabular}

Table B2

Statistical Significance: JMA Catalog—Poisson Reference

\begin{tabular}{lcrcccc}
\hline & $r$ & $M$ & $p_{r}$ & $p_{50}$ & $p_{90}$ & $\delta \lambda_{50}\left(\delta \lambda_{05,95}\right)[\%]$ \\
\hline All & 0.473 & 3958 & $\mathbf{2} \times \mathbf{1 0}^{-\mathbf{9}}$ & 0.50 & 0.05 & $\mathbf{1 8}(12,23)$ \\
All Distant & 0.496 & 2534 & 0.27 & 0.69 & 0.49 & $2(-3,8)$ \\
All Precursory & 0.497 & 2090 & 0.32 & 0.93 & 0.90 & $2(-4,8)$ \\
All Subsequent & 0.494 & 444 & 0.33 & 0.21 & 0.16 & $4(-9,18)$ \\
& & & & & & \\
\hline
\end{tabular}


Table C1

ISC Catalog

\begin{tabular}{lrccccc}
\hline \multicolumn{1}{c}{ Region } & $\mu$ [yr $^{-1}$ ] & $c$ [days] & $p$ & Fraction Aftershocks & Frac. Direct Aftershocks & Num. Local Mainshocks* \\
\hline Balleny Is. & 0.4 & 0.14 & 1.29 & 0.82 & 0.82 & 0 \\
New Ireland & 13.9 & 0.07 & 0.99 & 0.40 & 0.34 & 1 \\
Peru & 7.5 & 0.70 & 1.71 & 0.65 & 0.65 & 0 \\
Tokachi-Oki & 7.9 & 0.03 & 0.98 & 0.66 & 0.55 & 2 \\
Macquarie Is. & 0.1 & 0.01 & 1.53 & 0.67 & 0.67 & 0 \\
Sumatra-Andaman & 8.7 & 0.03 & 0.81 & 0.87 & 0.28 & 2 \\
Sumatra-Nias & 3.3 & 0.09 & 0.89 & 0.88 & 0.71 & 4 \\
Tonga & 18.5 & 0.71 & 1.26 & 0.52 & 0.44 & 5 \\
Kuril Is. & 13.6 & 0.08 & 0.97 & 0.80 & 0.44 & 0 \\
Kuril Is. & 1.6 & 0.63 & 1.52 & 0.95 & 0.29 & 5 \\
Solomon Is. & 5.8 & 1.00 & 1.60 & 0.82 & 0.32 & 3 \\
Central Peru & 5.9 & 0.13 & 1.17 & 0.72 & 0.70 & 0 \\
So. Sumatra & 2.8 & 0.04 & 0.90 & 0.89 & 0.56 & 0 \\
Samoa & 14.2 & 0.09 & 1.06 & 0.38 & 0.38 & 5 \\
Chile & 6.5 & 0.43 & 1.07 & 0.86 & 0.86 & 0.00 \\
Tohoku-Oki ${ }^{\dagger}$ & 17.9 & 0.01 & 0.83 & 0.56 & & \\
\hline
\end{tabular}

*Local mainshocks with $M<8$ only.

'Does not include the local $M \geq 8$ earthquake itself due to catalog time limit.

Table C2

PDE Catalog

\begin{tabular}{|c|c|c|c|c|c|c|}
\hline Region & $\mu\left[\mathrm{yr}^{-1}\right]$ & $c$ [days] & $p$ & Fraction Aftershocks & Frac. Direct Aftershocks & Num. Local Mainshocks* \\
\hline Balleny Is. & 0.1 & - & - & - & - & 0 \\
\hline New Ireland & 7.9 & 0.03 & 0.93 & 0.40 & 0.36 & 1 \\
\hline Peru & 2.2 & 0.45 & 1.64 & 0.63 & 0.63 & 0 \\
\hline Tokachi-Oki & 4.3 & 0.03 & 0.96 & 0.71 & 0.59 & 4 \\
\hline Macquarie Is. & 0.1 & 0.17 & 2.00 & 0.75 & 0.75 & 0 \\
\hline Sumatra-Andaman & 2.6 & 0.05 & 0.83 & 0.92 & 0.30 & 4 \\
\hline Sumatra-Nias & 0.2 & 1.00 & 0.81 & 0.87 & 0.71 & 2 \\
\hline Tonga & 10.1 & 1.00 & 1.28 & 0.55 & 0.44 & 5 \\
\hline Kuril Is. & 4.0 & 0.03 & 0.99 & 0.74 & 0.37 & 3 \\
\hline Kuril Is. & 0.4 & 0.34 & 1.49 & 0.94 & 0.16 & 0 \\
\hline Solomon Is. & 3.4 & 0.40 & 1.38 & 0.78 & 0.35 & 5 \\
\hline Central Peru & 5.0 & 0.12 & 1.18 & 0.72 & 0.61 & 2 \\
\hline So. Sumatra & 0.8 & 0.02 & 0.82 & 0.92 & 0.63 & 2 \\
\hline Samoa & 1.0 & 0.02 & 1.15 & 0.35 & 0.35 & 0 \\
\hline Chile & 2.6 & 0.55 & 1.19 & 0.90 & 0.84 & 1 \\
\hline Tohoku-Oki & 8.8 & 0.33 & 0.91 & 0.84 & 0.76 & 4 \\
\hline
\end{tabular}

*Local mainshocks with $M<8$ only.

Table C3

JMA Catalog

\begin{tabular}{ccccccc}
\hline Region & $\mu$ yrr $\left.^{-1}\right]$ & $c$ [days] & $p$ & Fraction Aftershocks & Frac. Direct Aftershocks & Num. Local Mainshocks* \\
\hline Tokachi-Oki & 175 & 0.07 & 0.86 & 0.59 & 0.45 & 4 \\
Tohoku-Oki & 2037 & 0.05 & 0.94 & 0.20 & 0.00 & 2 \\
\hline
\end{tabular}

*Local mainshocks with $M<8$ only.

Does not include the local $M \geq 8$ earthquake itself because there are no subsequent triggers.

total fraction of the simulated catalog made up of aftershocks (local, regional, and target events), the fraction that is aftershocks of the target $M \geq 8$ earthquake itself and the number of local significant mainshocks used to fit the time series.

See Tables C1, C2, and C3.
Department of Earth and Planet. Sci.

University of California, Santa Cruz

1156 High Street

Santa Cruz, California 95064 